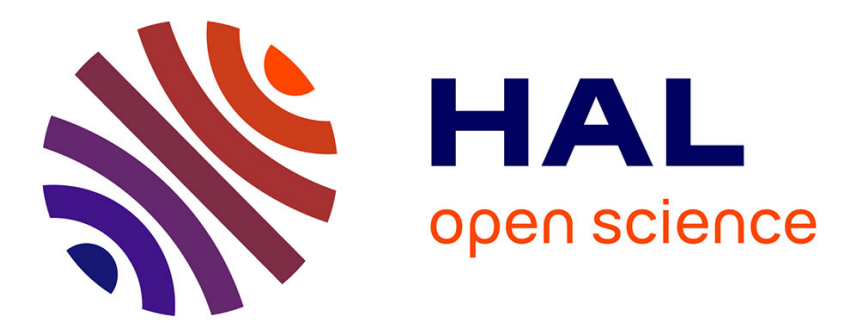

\title{
Construction of an aggregate consistent utility, without Pareto optimality. Application to Long-Term yield curve Modeling
}

\author{
Nicole El Karoui, Caroline Hillairet, Mohamed Mrad
}

\section{- To cite this version:}

Nicole El Karoui, Caroline Hillairet, Mohamed Mrad. Construction of an aggregate consistent utility, without Pareto optimality. Application to Long-Term yield curve Modeling. Frontiers in Stochastic Analysis - BSDEs, SPDEs and their Applications, 2019. hal-01721441

\author{
HAL Id: hal-01721441 \\ https://hal.science/hal-01721441
}

Submitted on 2 Mar 2018

HAL is a multi-disciplinary open access archive for the deposit and dissemination of scientific research documents, whether they are published or not. The documents may come from teaching and research institutions in France or abroad, or from public or private research centers.
L'archive ouverte pluridisciplinaire HAL, est destinée au dépôt et à la diffusion de documents scientifiques de niveau recherche, publiés ou non, émanant des établissements d'enseignement et de recherche français ou étrangers, des laboratoires publics ou privés. 


\title{
Construction of an aggregate consistent utility, without Pareto optimality. Application to Long-Term yield curve Modeling.
}

\author{
El Karoui Nicole, ${ }^{*} \quad$ Hillairet Caroline, ${ }^{\dagger}$ Mrad Mohamed $\ddagger$ \\ March 1, 2018
}

\begin{abstract}
The aim of this paper is to describe globally the behavior and preferences of heterogeneous agents. Our starting point is the global wealth of the economy, with a given repartition of the wealth among investors, which is not necessarily Pareto optimal. We propose a construction of an aggregate forward utility, market consistent, that aggregates the marginal utility of the heterogeneous agents. This construction is based on the aggregation of the pricing kernels of each investor. As an application we analyze the impact of the heterogeneity and of the global wealth market on the yield curve.
\end{abstract}

Keywords: Utility aggregation, heterogeneous preferences, market-consistent progressive utility, yield curve.

MSC 2010: 60H15, 91B16, 91B69.

\section{Introduction}

Most of general equilibrium macroeconomic models are simplified by assuming that consumers and/or firms could be described as a representative agent. That is agents may differ and act differently, but at equilibrium the sum of their choices is mathematically equivalent to the decision of one individual or many identical individuals. The way that preferences of multiple agents aggregate at equilibrium is a difficult task, and

*LPSM, UMR CNRS 8001, Sorbonne Université,

$\dagger$ EnsaeParisTech Crest, UMR CNRS 9194,

${ }^{\ddagger}$ LAGA, UMR CNRS 7539, Université Paris 13 
even if each individual preference is modeled by a simple function, it is unlikely that the aggregate utility could be reduced into a simple expression (unless all agents are identical). Heterogeneity of investors is an unavoidable feature that should be taken into account.

The literature on equilibrium risk sharing in complete markets with heterogeneous risk preferences starts with the seminal paper by Dumas [Dum89], with two agents with heterogeneous risk preferences. Chan and Kogan [CK02] consider an extension of the Wang [Wan96] model, with a continuum of agents with heterogeneous risk aversions. Yan [Yan10] and Jouini et al. [JN10] stress the impact of relative wealth fluctuations on the equilibrium characteristics. Cvitanic, Jouini et al. [CJMN11] were the first to propose an equilibrium model dealing with three types of heterogeneity: investors may differ in their beliefs, in their level of risk aversion and in their time-preference rate. They identify the channels through which heterogeneity impacts the different equilibrium characteristics. In their model, the aggregate parameters can be written as a risk tolerance weighted average of the individual parameters.

In the meantime, the existence of an equilibrium is not always satisfied and equilibrium are often stated and studied in a complete market setting. One key point for the existence of equilibrium is that agents agree on the same state price density process (also called pricing kernel), which is the same for all agents. However, if no equilibrium exists, is it still possible to propose a representative utility aggregating the preferences of all investors in the economy?

In this paper, we start from the weaker hypothesis of non arbitrage, and we consider an incomplete market, with given exogenous market parameters. Our aim is to propose a way of describing globally the behavior of heterogeneous agents investing in this market, heterogeneous by their preferences, their weights or sizes. To do this we construct a stochastic utility process corresponding to the aggregate wealth of the economy and to the aggregate pricing kernels. We do not deal with agents interactions, nor equilibrium, neither Pareto optimality: the repartition of the wealth among market investors is given. The global wealth of the whole economy is naturally defined by the aggregation of the wealth of all individuals. The problem consists then in deriving a utility process for which this global wealth is optimal. This is related to a calibration approach, and to do this the progressive framework is well adapted (see [KHM17a]). Besides, the progressive approach has also many advantages. First of all, it allows to model the change of the preferences of the investors along time. Indeed, in a dynamic and stochastic environment, the standard notion of utility function is not flexible enough to help us to make good choices in the long run. The utility criterion must be adaptative and adjusted to the information flow. Musiela and Zariphopoulou were the 
first to suggest to use instead of the classic criterion the concept of progressive dynamic utility, that gives an adaptive way to model possible changes over the time of individual preferences of an agent. Characterization of market-consistent progressive utility has been then studied in a general setting in El Karoui and Mrad [KM13, KM16b]. Secondly, the theoretical study of progressive utility emphasizes the dependency of the optimal processes with respect to their initial conditions. This dependency and some non linearity effects are illustrated in the example of the valuation of the discount rates. In the economic modeling, interest rates are determined endogenously at equilibrium, mainly in an economy composed of identical investors (see for example the well known Vasicek [Vas77] or Cox Ingersoll Ross [CIR85] models). In our framework, the market is incomplete and in place of the traditional (complete) pricing rule, we price the zerocoupon bonds using the indifference pricing rule, based on the marginal indifference pricing. A numerical example is proposed based on an extension of the Vasicek model of the yield curve.

The paper is organized as follow. First we define in Section 2 the investment universe and we recall the framework and the main properties of market consistent progressive utilities, and the characterization of a consistent utility from its optimal primal and dual processes. Section 3 states the main results about preferences aggregation: from the characteristics of the investors, we construct an aggregate consistent progressive utility process, by aggregating the wealth of each investors and their pricing kernels. To illustrate this theory, we give the example of aggregating power utilities. In particular we show that aggregating power utilities does not lead to a power utility, except if all investors share the same risk aversion. Thus taking a power utility for the representative agent, as it is done in many economic papers, assumes actually a very strong hypothesis of homogeneity of the different investors in the economy. Section 4 studies the impact of the heterogeneity of investors, that induces dependency and non-linearity in the valuation of financial assets. The particular example developed here consists in the valuation of discount rates and the impact of the global wealth on this rates. Some numerics illustrate the impact of the different parameters on the yield curve. Technical regularity conditions are postponed in the Appendix.

\section{Investment universe and Consistent progressive utility.}

\subsection{The investment universe}

Let us consider an incomplete Itô market, defined on a filtered probability space $\left(\Omega,\left(\mathcal{F}_{t}\right), \mathbb{P}\right)$ (satisfying usual condition of completion and right continuity) driven by 
a $n$-standard Brownian motion $W$. As usual, the market is characterized by some exogenous progressive processes: the short rate $\left(r_{t}\right)$ and a $n$-dimensional risk premium $\left(\eta_{t}\right)$, satisfying the integrability condition $\int_{0}^{T}\left(r_{s}+\left|\eta_{s}\right|^{2}\right) d s<\infty$ for any $T$. The agent may invest in this financial market. To be short, we give the mathematical definition of the class of admissible strategies ${ }^{1}\left(\kappa_{t}\right)$, without specifying the risky assets. The incompleteness of the market is expressed by restrictions on the risky portfolios $\kappa_{t}$ constrained to live in a given progressive vector space $\mathcal{R}_{t}$. To fix the idea, if the incompleteness follows only from the fact that the number of assets is less than the dimension $n$ of the Brownian motion, then typically $\mathcal{R}_{t}=\sigma_{t}\left(\mathbb{R}^{n}\right)$. For an Itô market, good references are Karatzas, Lehoczky, Shreve [KLS87] or the book of Karatzas and Shreve [KS01], and in a more general context Kramkov, Schachermayer [KS03].

To avoid technicalities, we assume throughout the paper that all the processes satisfy the necessary (progressive) measurability and integrability conditions such that the following formal manipulations and statements are meaningful. The following short notations will be used extensively. Let $\mathcal{R}$ be a vector subspace of $\mathbb{R}^{n}$. For any $x \in \mathbb{R}^{n}$, $x^{\mathcal{R}}$ is the orthogonal projection of the vector $x$ onto $\mathcal{R}$ and $x^{\perp}$ is the orthogonal projection onto $\mathcal{R}^{\perp}$.

Definition 2.1 (Admissible portfolio). (i) The self-financing dynamics of a wealth process with risky portfolio $\kappa$, starting from the initial wealth $x>0$, is given by

$$
d X_{t}^{\kappa}=X_{t}^{\kappa}\left[r_{t} d t+\kappa_{t}\left(d W_{t}+\eta_{t} d t\right)\right], \quad \kappa_{t} \in \mathcal{R}_{t}, \quad \text { and } \quad X_{0}^{\kappa}=x
$$

where $\kappa$ is a progressive $n$-dimensional vector measuring the volatility vector of the wealth $X^{\kappa}$, such that $\int_{0}^{T}\left\|\kappa_{t}\right\|^{2} d t<\infty$, a.s..

(ii) A self-financing strategy $\left(\kappa_{t}\right)$ is admissible if the portfolio $\kappa$ lives in a given progressive family of vector spaces $\left(\mathcal{R}_{t}\right)$ a.s..

(iii) The set of the wealth processes with admissible $\left(\kappa_{t}\right)$ (called admissible wealth processes) starting from the initial wealth $x$ is denoted by $\mathscr{X}(x)$, and $\mathscr{X}$ when the initial wealth is not specified.

The existence of a risk premium $\eta$ formulates the absence of arbitrage opportunity. Since from (2.1), the impact of the risk premium on the wealth dynamics only appears through the term $\kappa_{t} \cdot \eta_{t}$ for $\kappa_{t} \in \mathcal{R}_{t}$, there is a "minimal" risk premium $\left(\eta_{t}^{\mathcal{R}}\right)$, the projection of $\eta_{t}$ on the space $\mathcal{R}_{t}\left(\kappa_{t} \cdot \eta_{t}=\kappa_{t} \cdot \eta_{t}^{\mathcal{R}}\right)$, to which we refer in the sequel. In the following definition, we are interested in the class of the so-called state price density processes $Y^{\nu}$ (taking into account the discount factor) which are also called the pricing kernels.

\footnotetext{
${ }^{1} \kappa_{t}=\sigma_{t} \pi_{t}$ with $\pi$ being the fraction of wealth invested in the risky assets, and $\sigma$ being the volatility process.
} 
Definition 2.2 (State price density process). A positive Itô semimartingale $Y^{\nu}$ is called an admissible state price density process if for any admissible wealth process $X^{\kappa} \in \mathscr{X}$,

$$
X_{t}^{\kappa} Y_{t}^{\nu} \quad \text { is a local martingale. }
$$

The simplest example of such process is the market state price process $Y^{0}\left(\nu=0, Y_{0}^{0}=\right.$ 1). In particular $\left(X_{t}^{\kappa} Y_{t}^{0}\right)$ is a local martingale, whose volatility $\left(\kappa_{t}-\eta_{t}^{\mathcal{R}}\right)$ belongs to $\mathcal{R}_{t}$. The martingale property (2.2) can be then expressed in terms of the ratio $\left(L_{t}^{\nu}=Y_{t}^{\nu} / Y_{t}^{0}\right)$ as $\left(X_{t}^{\kappa} Y_{t}^{0} L_{t}^{\nu}\right)$ is a local martingale or equivalently $\left(L_{t}^{\nu}\right)$ is a local exponential martingale whose volatility belongs to $\mathcal{R}_{t}^{\perp}$.

Corollary 2.3. Denote $\mathscr{Y}(y)$ the convex family of all admissible state density processes $Y^{\nu}(y)$ issued from $y$, and $\mathscr{Y}$ the set of all $\mathscr{Y}(y)$. Any $Y^{\nu}(y)$ is the product of the market state price process $Y^{0}$ by an exponential martingale $L^{\nu}(y)$ whose volatility $\nu$ belongs to $\mathcal{R}^{\perp}$. The differential decomposition of these three processes is

$$
\left\{\begin{aligned}
d Y_{t}^{0} & =Y_{t}^{0}\left[-r_{t} d t-\eta_{t}^{\mathcal{R}} \cdot d W_{t}\right], \quad Y_{0}^{0}=1 \\
d L_{t}^{\nu} & =L_{t}^{\nu}\left[\nu_{t} \cdot d W_{t}\right], \quad \nu_{t} \in \mathcal{R}_{t}^{\perp} \quad L_{0}^{\nu}=y \\
d Y_{t}^{\nu} & =Y_{t}^{\nu}\left[-r_{t} d t+\left(\nu_{t}-\eta_{t}^{\mathcal{R}}\right) \cdot d W_{t}\right], \quad \nu \in \mathcal{R}_{t}^{\perp} \quad Y_{0}^{\nu}=y .
\end{aligned}\right.
$$

Interesting discussions on the links between the state price density processes and the admissible market numeraire $1 / Y_{t}^{0}$, also called GOP (growth optimal portfolio) can be found in Geman, El Karoui, Rochet [KGR95], in Heath, Platen book [PH06], and in Filipovic, Platen [FP09]. Besides, the state price density processes are also called "pricing kernels" since they are useful for evaluating contingent claims under the historical probability measure $\mathbb{P}$. Not surprisingly, we will focus on them in the application of Section 4 about the valuation of zero-coupon bond and the modeling of the yield curve.

\subsection{Consistent progressive utility and their characteristics}

The preferences of the agents investing in the financial market are modeled by consistent progressive utility. The sub-cone of admissible wealth processes $\mathscr{X}$, describing the financial landscape, is considered in this forward setting as a family of test processes. As in statistical learning, the utility criteria are dynamically adjusted to this given family of test processes, also called the learning set. The time-coherence is then obtained from a dynamic decision criterion adjusted progressively over the time to this set $\mathscr{X}$.

More precisely, a progressive utility $\mathbf{U}$ is defined as a family of càdlàg adapted processes $\left(U(t, x), x \in \mathbb{R}^{+}\right)$such that $\mathbb{P}$.a.s., for every $t \geq 0$, the functions $x \in \mathbb{R}^{+} \mapsto$ $U(t, x, \omega)$ are standard utility functions. As usual, a utility function $u$ is a strictly concave, strictly increasing, and non-negative function defined on $\mathbb{R}^{+}$, with continuous marginal utility the derivative $u_{x}$, satisfying the Inada conditions $\lim _{x \mapsto \infty} u_{x}(x)=0$ 
and $\lim _{x \mapsto 0} u_{x}(x)=\infty$. The risk aversion coefficient $\mathrm{R}_{\mathrm{A}}(u)$ is measured by the ratio $\mathrm{R}_{\mathrm{A}}(u)(x)=-u_{x x}(x) / u_{x}(x)$ and the relative risk aversion by $\mathrm{R}_{\mathrm{A}}^{\mathrm{r}}(u)(x)=x \mathrm{R}_{\mathrm{A}}(u)(x)$. The asymptotic elasticity $E_{A}(u)(x)=\limsup _{x \mapsto \infty} x u_{x}(x) / u(x)$ is a key parameter in the optimization problem (see Kramkov [KS99]). As usual, the dual problem is based on the Fenchel-Legendre convex conjugate transformation $\tilde{u}(y)$ of a utility function $u$, where $\tilde{u}$ satisfies $\tilde{u}(y)=\sup _{x>0}(u(x)-y x)$. In particular, $\tilde{u}(y) \geq u(x)-y x$ and the maximum is attained at $u_{x}(x)=y$. Under Inada conditions, $\tilde{u}$ is twice continuously differentiable, strictly convex, strictly decreasing, with $\tilde{u}\left(0^{+}\right)=u(+\infty), \tilde{u}(+\infty)=u\left(0^{+}\right)$, a.s. Moreover, the marginal utility $u_{x}$ is the inverse of the opposite of the marginal conjugate utility $\tilde{u}_{y}$; that is $u_{x}^{-1}(y)=-\tilde{u}_{y}(y) ; \tilde{u}(y)=u(-\tilde{u}(y))+\tilde{u}_{y}(y) y$, and $u(x)=$ $\tilde{u}\left(u_{x}(x)\right)+x u_{x}(x)$.

Throughout the paper, we adopt the convention of small letters for deterministic utilities and capital letters for stochastic utilities.

\subsubsection{Characteristics of the consistent progressive utility}

The progressive utilities are adjusted to the learning set $\mathscr{X}$. The satisfaction provided by a test process $X^{\kappa} \in \mathscr{X}$ is measured by the dynamic criterion $\left(U\left(t, X_{t}^{\kappa}\right)\right)$. Since $\mathscr{X}$ is a learning set, there is no satisfaction to invest in the set $\mathscr{X}$, in other words in mean the future is less preferable than the present. From the mathematical point of view, this is equivalent to the supermartingale property of the dynamic preference process $\left(U\left(t, X_{t}^{\kappa}\right)\right)$. Moreover, to ensure that the stochastic utility $(U(t, x))$ is optimally adjusted, we make the additional assumption that the previous supermartingale constraint is binded by some optimal process $\kappa^{*}$ whose preference criterion $\left(U\left(t, X_{t}^{\kappa^{*}}\right)\right)$ is a martingale.

Definition 2.4 (Consistent progressive utility). Let $\mathbf{U}$ be a progressive utility with learning set $\mathscr{X}$.

(i) The utility $\mathbf{U}$ is said to be $\mathscr{X}$-consistent, if for any admissible test process $X^{\kappa} \in \mathscr{X}$, the preference process $\left(U\left(t, X_{t}^{\kappa}\right)\right)$ is a non-negative supermartingale.

(ii) The consistent utility $\mathbf{U}$ is said to be $\mathscr{X}$-strongly consistent if there exists an optimal process $X^{*}:=X^{\kappa^{*}} \in \mathscr{X}$, with $\kappa_{t}^{*} \in \mathcal{R}_{t}$, binding the constraint, in the sense that the optimal preference process $\left(U\left(t, X_{t}^{*}\right)\right)$ is a martingale.

The value function $(\mathcal{U}(t, x))$ of the classical optimization problem is an example of strongly $\mathscr{X}$-consistent utility, defined from its terminal condition $\mathcal{U}\left(T_{H}, x\right)=u(x)$ (see [KHM17a] for a general discussion between the forward and the backward viewpoints of utility functions).

The consistency property of the progressive utility $\mathbf{U}$ has a natural equivalent for dual progressive utility, as stated in the following proposition (see [KM13] for the proof). 
Proposition 2.5. $\mathrm{U}$ is a consistent progressive utility with the class $\mathscr{X}$ if and only if its Fenchel transform $\widetilde{\mathbf{U}}$ is consistent with the class $\mathcal{Y}$ in the sense that $\widetilde{U}\left(t, Y_{t}\right)$ is a submartingale for any $Y \in \mathcal{Y}$, and there exists some $Y^{*} \in \mathcal{Y}$ (called dual optimal process) such that $\widetilde{U}\left(t, Y_{t}^{*}\right)$ is a martingale. Moreover, the two optimal processes are related by the main identity $U_{x}\left(t, X_{t}^{*}(x)\right)=Y_{t}^{*}\left(u_{x}(x)\right)$.

Rogers provides in [Rog03] a unified (and very simple) approach to get very quickly a simple heuristic of the main identity $U_{x}\left(t, X_{t}^{*}(x)\right)=Y_{t}^{*}\left(u_{x}(x)\right)$, that will be at the cornerstone of this paper.

\section{LOCAL CHARACTERISTICS OF CONSISTENT FORWARD UTILITY}

The "global" supermartingale property implied by the consistency condition may be transfered into local conditions on the differential characteristics of the utility process U. El Karoui and Mrad [KM13] obtained a non linear HJB-SPDE under the general assumption that the utility random field $\mathbf{U}$ is a "regular" Itô random field with differential decomposition,

$$
d U(t, x)=\beta(t, x) d t+\gamma(t, x) \cdot d W_{t},
$$

where $\beta(t, x)$ is the drift random field and $\gamma(t, x)$ is the multivariate diffusion random field. The regularity assumption recalled in the Appendix, allows in particular to use the Itô-Ventzel formula and to show that the marginal utility $\left(U_{x}(t, x)\right)$ is also an Itô random field with local characteristics $\left(\beta_{x}(t, x), \gamma_{x}(t, x)\right)$. We give the main result about the consistency characterization through a HJB contraint:

Theorem 2.6 (Consistency). Let $\mathbf{U}$ be a "regular utility" system and $(\beta, \gamma)$ its local characteristics. The utility random field $\mathbf{U}$ is strongly consistent with the family of test processes $\mathscr{X}=\left\{X^{\kappa}, \mid \kappa \in \mathcal{R}\right\}$ if and only if $(a)$ and $(b)$ holds :

(i) a) The drift random field $\beta$ satisfies the HJB-constraint, $d \mathbb{P} \times d t . a . s$.

$$
\left\{\begin{aligned}
\beta(t, x) & =-U_{x}(t, x) r_{t} x-\frac{1}{2} \sup _{\sigma \in \mathcal{R}}\left\{U_{x x}(t, x)\left(\left\|\sigma_{t}\right\|^{2}+2 \sigma_{t} \cdot\left(\frac{U_{x}(t, x) \eta_{t}^{\mathcal{R}}+\gamma_{x}(t, x)}{U_{x x}(t, x)}\right)\right)\right\} . \\
& \left.=-U_{x}(t, x) x r_{t}+\frac{1}{2 U_{x x}(t, x)} \| U_{x}(t, x) \eta_{t}^{\mathcal{R}}+\gamma_{x}^{\mathcal{R}}(t, x)\right) \|^{2} . \\
& \left.=-U_{x}(t, x) x r_{t}+\frac{1}{2} U_{x x}(t, x) \| \sigma^{*}(t, x)\right) \|^{2}
\end{aligned}\right.
$$

The quantity $\frac{\gamma_{x}^{\mathcal{R}}(t, x)}{U_{x}(t, x)}$ can be interpreted as an "utility risk premium".

b) The stochastic differential equation $S D E^{\mathcal{R}}\left(\sigma^{*}\right)$

$$
\begin{cases}d X_{t}^{*} & =r_{t} X_{t}^{*} d t+\sigma^{*}\left(t, X_{t}^{*}\right)\left(d W_{t}+\eta_{t}^{\mathcal{R}} d t\right), \\ \sigma^{*}(t, x) & =-\frac{U_{x}(t, x)}{U_{x x}(t, x)}\left(\eta_{t}^{\mathcal{R}}+\frac{\gamma_{x}^{\mathcal{R}}(t, x)}{U_{x}(t, x)}\right)=x \kappa^{*}(t, x)\end{cases}
$$

admits a strong solution $X^{*}$, which is an optimal portfolio in the preference sense. 
(ii) In addition, the positive process $U_{x}\left(t, X_{t}^{*}(x)\right)$ is the optimal dual state price process $Y_{t}^{*}\left(u_{x}(x)\right)$, solution of the $S D E^{\perp}\left(\vartheta^{*, \perp}\right)$ issued from $y=u_{x}(x)$

$$
\left\{\begin{array}{l}
d Y_{t}^{*}=-r_{t} Y_{t}^{*} d t+\left(\vartheta^{*, \perp}\left(t, Y_{t}^{*}\right)-\eta_{t}^{\mathcal{R}} Y_{t}^{*}\right) \cdot d W_{t} \\
\vartheta^{*, \perp}(t, y)=\gamma_{x}^{\perp}\left(t, U_{x}^{-1}(t, y)\right)=y \nu^{*, \perp}(t, y) .
\end{array}\right.
$$

The regularity assumptions on $\mathbf{U}$ recalled in the Appendix imply that the coefficients of the SDEs (2.6) and (2.7) are regular enough to ensure that $X^{*}$ and $Y^{*}$ are monotonic (increasing) with respect to their respective initial condition $x$ and $y$ with range $[0, \infty]$ (see [KM13]).

\subsubsection{Consistent power utility and separability}

Power utilities with constant relative risk aversion $\theta \in] 0,1\left[, u^{(\theta)}(x)=\frac{x^{1-\theta}}{1-\theta}\right.$ are the standard framework in the economic literature, useful for its simplicity and the easy interpretation of the parameters. In particular, the parameter $\theta$ is the relative risk aversion coefficient $\mathrm{R}_{\mathrm{A}}^{\mathrm{r}}\left(u^{(\theta)}\right)(x)=-x u_{x x}^{(\theta)}(x) / u_{x}^{(\theta)}(x)=\theta$.

Consistent progressive power utilities $U^{(\theta)}(t, x)$ are the product of their initial condition $u^{(\theta)}(x)$ by a coefficient $Z_{t}^{(\theta)}$. Despite their stochastic structure, their relative risk aversion coefficients are still constants, $\mathrm{R}_{\mathrm{A}}^{\mathrm{r}}\left(U^{(\theta)}\right)(t, x)=\mathrm{R}_{\mathrm{A}}^{\mathrm{r}}(u)(x)=\theta$.

The role of the stochastic process $Z_{t}^{(\theta)}$ is to guarantee the market consistency of dynamics power utility. Since $u_{x}^{(\theta)}(1)=1$, the process $Z_{t}^{(\theta)}$, we have $Z_{t}^{(\theta)}=U_{x}^{(\theta)}(t, 1)$. Since $U^{(\theta)}=Z^{(\theta)} u^{(\theta)}$, its local characteristics $\left(\beta^{(\theta)}, \gamma^{(\theta)}\right)$ are proportional to $u^{(\theta)}$, with $\beta^{(\theta)}(t, x)=\mu_{t}^{(\theta)} u^{(\theta)}(x)$ and $\gamma^{(\theta)}(t, x)=Z_{t}^{(\theta)} \delta_{t}^{(\theta)} u^{(\theta)}(x),\left(\mu_{t}^{(\theta)}, Z_{t}^{(\theta)} \delta_{t}^{(\theta)}\right)$ being the stochastic parameters of the semimartingale $Z^{(\theta)}$. Theorem 2.6 characterizes the optimal processes of power progressive utilities.

Proposition 2.7. Let $\left(U^{(\theta)}(t, x)=Z_{t}^{(\theta)} u^{(\theta)}(x)\right)$ be a power consistent progressive utility, $\left(Z_{t}^{(\theta)}\right)$ being a positive semimartingale with parameters $\left(\mu_{t}^{(\theta)}, Z_{t}^{(\theta)} \delta_{t}^{(\theta)}\right)$.

(i) The optimal processes $X_{t}^{(*, \theta)}(x)$ and $Y_{t}^{(*, \theta)}(y)$ are linear with respect to their initial conditions, $X^{(*, \theta)}(x)=x \bar{X}^{(*, \theta)}$ and $Y^{(*, \theta)}(x)=y \bar{Y}^{(*, \theta)}$, with dynamics

$$
\left\{\begin{array}{l}
d \bar{X}_{t}^{(*, \theta)}=\bar{X}_{t}^{(*, \theta)}\left[r_{t}+\frac{1}{\theta}\left(\eta_{t}^{\mathcal{R}}+\delta_{t}^{(\theta), \mathcal{R}}\right) \cdot\left(d W_{t}+\eta_{t}^{\mathcal{R}} d t\right)\right] \\
d \bar{Y}_{t}^{(*, \theta)}=\bar{Y}_{t}^{(*, \theta)}\left[-r_{t} d t+\left(\delta_{t}^{(\theta), \perp}-\eta_{t}^{\mathcal{R}}\right) \cdot d W_{t}\right]
\end{array}\right.
$$

The coefficient $\delta_{t}^{(\theta), \mathcal{R}}$ describes how the stochasticity of the utility influences the investment strategy $\kappa_{t}^{(*, \theta)}=\frac{1}{\theta}\left(\eta_{t}^{\mathcal{R}}+\delta_{t}^{(\theta), \mathcal{R}}\right)$.

(ii) The drift of the process $\left(Z_{t}^{(\theta)}\right)$ is not free, since the consistency condition (equivalent to the HJB constraint) implies that

$$
-Z_{t}^{(\theta)}=\left[\bar{X}_{t}^{(*, \theta)}\right]^{\theta} \bar{Y}_{t}^{(*, \theta)} \text {, and }
$$


$-\mu_{t}^{(\theta)}=-(1-\theta) Z_{t}^{(\theta)}\left(r_{t}+\frac{1}{2 \theta}\left\|\eta_{t}^{\mathcal{R}}+\delta_{t}^{(\theta), \mathcal{R}}\right\|^{2}\right)$.

The consistent power utilities are completely specified by the volatility $\left(\delta_{t}^{(\theta)}\right)$ of the $d y$ namics coefficient $Z_{t}^{(\theta)}$.

Proof. (i) By Equation (2.6), the volatility of the optimal process $X_{t}^{(*, \theta)}(x)$ is linear with respect to the initial wealth $x, \sigma^{(*, \theta)}(t, x)=\frac{x}{\theta}\left(\eta_{t}^{\mathcal{R}}+\delta_{t}^{(\theta), \mathcal{R}}\right)$. Since the drift is also linear, the optimal process is linear with respect to the initial wealth, $X^{(*, \theta)}(x)=x \bar{X}^{(*, \theta)}$ where the dynamics of $\bar{X}^{(*, \theta)}$ is given by Equation (2.8). The dual process $Y_{t}^{(*, \theta)}(y)$ is also linear with respect to $y$, and by Equation $(2.7), \vartheta^{(*, \theta)}(t, y)=y \delta_{t}^{(\theta), \perp}$. Then, the dynamic of $\left(\bar{Y}_{t}^{(*, \theta)}\right)$ is given by Equation (2.8).

(ii) By the optimality relation, $U_{x}^{(\theta)}\left(t, X^{(*, \theta)}(t, x)\right)=u_{x}(x) Y_{t}^{(*, \theta)}$. This property is equivalent to the HJB constraint on the $\operatorname{drift} \beta^{(\theta)}(t, x)=\mu_{t}^{(\theta)} u^{(\theta)}(x)$ of the power utility. A consequence is that $Z_{t}^{(\theta)}=\left[\bar{X}_{t}^{(*, \theta)}\right]^{\theta} \bar{Y}_{t}^{(*, \theta)}$. The linearity of the different processes yields

$$
d Z_{t}^{(\theta)}=Z_{t}^{(\theta)}\left[-\left((1-\theta) r_{t}+\frac{1-\theta}{2 \theta}\left\|\eta_{t}^{\mathcal{R}}+\delta_{t}^{(\theta), \mathcal{R}}\right\|^{2}\right) d t+\delta_{t}^{(\theta)} \cdot d W_{t}\right] .
$$

The drift of $Z^{(\theta)}$ depends only of the market parameters $\left(r_{t}, \eta_{t}^{\mathcal{R}}\right)$ and its volatility $\delta_{t}^{(\theta)}$.

Remark 2.1. Power utilities have also the remarkable property to be the only consistent separable progressive utilities $U(t, x)=Z_{t} u(x)$. The HJB equation (2.5) leads to a contradiction as soon as the functions $\phi^{1}=x u_{x} / u$ and $\phi^{2}=x u_{x x} / u_{x}$ are not constant, since the HJB constraint on the drift $\beta(t, x)$ implies that the time function $\rho_{t}^{Z}$ satisfies $\rho_{t}^{Z}=-\phi^{1}(x) r_{t}+\phi^{1}(x) / \phi^{2}(x)\left\|\eta_{t}^{\mathcal{R}}+\delta_{t}^{Z, \mathcal{R}}\right\|^{2}$ for any $x$. An exception is given by the case where $\delta_{t}^{Z, \mathcal{R}}=-\eta_{t}^{\mathcal{R}}$ and $r_{t}=0$. In this case, $Z_{t}$ is an exponential martingale with volatility $\eta_{t}^{\mathcal{R}}$ multiplied by an orthogonal exponential martingale with volatility $\delta_{t}^{Z, \perp}$.

\subsection{Reverse Problem}

One remarkable feature proved in [KM13] is that properties given in Theorem 2.6 are in fact necessary and sufficient conditions to reconstruct a consistent progressive utility from two optimal processes $X^{*}$ et $Y^{*}$, when theses processes are monotonic with respect to their initial condition. This construction relies on the identity $U_{x}\left(t, X_{t}^{*}(x)\right)=Y_{t}^{*}\left(u_{x}(x)\right)$, using monotonicity and regularity of optimal random fields, and some integrability condition near zero of the initial utility.

Let us consider two increasing monotonic processes $X^{*}(x) \in \mathscr{X}(x)$ and $Y^{*}(y) \in \mathscr{Y}(y)$, strong regular solutions of the two SDEs

$$
\left\{\begin{array}{l}
d X_{t}^{*}=r_{t} X_{t}^{*} d t+\sigma^{*}\left(t, X_{t}^{*}\right)\left(d W_{t}+\eta_{t}^{\mathcal{R}}\right) d t, \quad X_{0}^{*}=x \\
d Y_{t}^{*}=-r_{t} Y_{t}^{*} d t+\left(\vartheta^{*, \perp}\left(t, Y_{t}^{*}\right)-\eta_{t}^{\mathcal{R}} Y_{t}^{*}\right) \cdot d W_{t}, \quad Y_{0}^{*}=y
\end{array}\right.
$$


The dynamics of $X^{*} \in \mathscr{X}$ is uniquely determined by its diffusion coefficient $\sigma^{*} \in \mathcal{R}$; the corresponding $\operatorname{SDE}$ is denoted $\operatorname{SDE}^{\mathcal{R}}\left(\sigma^{*}\right)$. Similarly the dynamics of $Y^{*} \in \mathscr{Y}$ is uniquely determined by its diffusion coefficient $\vartheta^{*, \perp} \in \mathcal{R}^{\perp}$; the corresponding SDE is denoted $\operatorname{SDE}^{\perp}\left(\vartheta^{*, \perp}\right)$.

We now give sufficient conditions on the coefficients $\sigma^{*}(t, x)$ and $\vartheta^{*, \perp}(t, y)$ which ensure on the one hand the monotonicity of the solutions of Equations (2.9) and the semimartingale decomposition of the random field $\mathcal{X}^{*}$ the inverse flow of $X^{*}$; and on the other hand that the random field $\mathbf{V}$ defined by $V(t, x):=Y_{t}^{*}\left(u_{x}\left(\mathcal{X}^{*}(t, x)\right)\right)$ is the derivative of a progressive utility $\mathbf{U}$. The sufficient regularity conditions we state below are proved in [KM13].

\subsubsection{Technical results}

In this presentation we clearly favor the SDE point of view for the processes $X^{*}$ and $Y^{*}$. This allows us to use the existing results in SDE's theory and provide sufficient regularity conditions $\left(\overline{\mathcal{K}}_{\mathbf{b}}^{0,1} \cap \overline{\mathcal{K}}_{\text {loc }}^{3, \delta}\right)^{2}$ on the coefficients to ensure the existence of regular SDE solutions. Global Lipschitz condition $\left(\overline{\mathcal{K}}_{\mathbf{b}}^{0,1}\right)$ is enough to obtain strong and monotonic solutions whereas the regularity is ensured by the local conditions $\left(\overline{\mathcal{K}}_{l o c}^{m, \delta}\right)$. But this point of view is not necessary as soon as one starts from non-explosive monotonic solutions $X^{*}$ and $Y^{*}$. We first recall the present version of some results in [KM13].

Proposition 2.8 (Regularity). Let us consider the two stochastic equations $\operatorname{SDE}^{\mathcal{R}}\left(\sigma^{*}\right)$ and $\operatorname{SDE}^{\perp}\left(\vartheta^{*, \perp}\right)$ defined in (2.9) and assume

$$
\sigma^{*} \in \overline{\mathcal{K}}_{\mathbf{b}}^{0,1} \cap \overline{\mathcal{K}}_{\text {loc }}^{3, \delta} \text {, and } \quad \vartheta^{*, \perp} \in \overline{\mathcal{K}}_{\mathbf{b}}^{0,1} \cap \overline{\mathcal{K}}_{\text {loc }}^{2, \delta} \quad \text { for some } \delta \in(0,1] .
$$

(i) Then, the differential equations $\operatorname{SDE}^{\perp}\left(\vartheta^{*, \perp}\right)$ and $\operatorname{SDE}^{\mathcal{R}}\left(\sigma^{*}\right)$ admit two regular monotonic solutions $Y^{*}$ and $X^{*}$ with different regularity.

- The solution $Y^{*}$ belongs to $\mathcal{K}_{\text {loc }}^{2, \varepsilon}$, and its diffusion local characteristic $\psi(., y):=$ $\vartheta^{*, \perp}\left(., Y^{*}(y)\right)$ is in $\overline{\mathcal{K}}_{\text {loc }}^{2, \varepsilon}$ for all $\varepsilon \in[0, \delta[$.

- The solution $X^{*}$ belongs to $\mathcal{K}_{\text {loc }}^{3, \varepsilon}$ and its diffusion local characteristics $\phi(., x):=$ $\sigma^{*}\left(., X^{*}(x)\right)$ is in $\overline{\mathcal{K}}_{\text {loc }}^{3, \varepsilon}$ for all $\varepsilon \in[0, \delta[$.

(ii) The range of the maps $x \mapsto X^{*}(x)$ and $y \mapsto Y^{*}(y)$ is $] 0,+\infty\left[\right.$. The inverse $\mathcal{X}^{*}$ of $X^{*}$ is a semimartingale, unique monotonic solution of the stochastic PDE,

$$
\begin{cases}d \mathcal{X}^{*}(t, x) & =-\mathcal{X}_{x}^{*}(t, x)\left[\sigma^{*}(t, x) \cdot\left(d W_{t}+\eta_{t}^{\mathcal{R}} d t\right)+r_{t} x d t\right]+\widehat{L}_{t, x}^{*}(\mathcal{X}) d t \\ \widehat{L}_{t, x}^{*} & :=\frac{1}{2} \partial_{x}\left(\left\|\sigma^{*}(t, x)\right\|^{2} \partial_{x}\right) .\end{cases}
$$

\footnotetext{
${ }^{2}$ See the Appendix for the definition of this classes of regularity.
} 
For integrability reasons, we need to control the speed of convergence of $X^{*}$ and $Y^{*}$ at 0 and $\infty$. The following results are standard under Lipschitz conditions, satisfied in our setting, see [Kun97].

Corollary 2.9. The asymptotic behaviors of $X^{*}$ and $Y^{*}$ are similar and well-controlled in time. The short notation $\max \left(Z_{T}(z)\right)=\sup _{0 \leq t \leq T} Z(t, z)$ is used in the sequel. More precisely, if $Z$ is one of the two processes $X^{*}$ and $Y^{*}$, for any $T$ almost surely, for any $\varepsilon \in(0,1)$, uniformly on $[0, T]$, the asymptotic limits in $\infty$ or 0 are,

$$
\left\{\begin{array}{l}
\lim _{z \rightarrow+\infty}\left(z^{-(1+\varepsilon)} \max \left(Z_{T}(z)\right)\right)=0 \text { and } \lim _{z \rightarrow+\infty}\left(z^{-\varepsilon} \max \left(Z_{T}(z)\right)\right)=\infty, \\
\lim _{z \rightarrow 0}\left(z^{-\varepsilon} \max \left(Z_{T}(z)\right)\right)=0 \text { and } \lim _{z \rightarrow 0}\left(z^{-(1+\varepsilon)} \max \left(Z_{T}(z)\right)\right)=\infty
\end{array}\right.
$$

Sometimes, it is more interesting to consider SDE's solutions as random fields $X^{*}(t, x)$ or $Y^{*}(t, y)$ with local characteristics $\phi^{*}(t, x)=\sigma^{*}\left(t, X_{t}^{*}(x)\right)$ or $\psi^{*}(t, y)=\vartheta^{*, \perp}\left(t, Y_{t}^{*}(y)\right)$. With the random fields point of view, non negativity and monotonicity are not so easy to prove.

Corollary 2.10. Let $\left(X_{t}^{*}(x)\right)$ and $\left(Y_{t}^{*}(y)\right)$ be two monotonic random fields,

$$
\left\{\begin{array}{l}
d X_{t}^{*}(x)=r_{t} X_{t}^{*}(x) d t+\phi^{*}(t, x)\left(d W_{t}+\eta_{t}^{\mathcal{R}}\right), \quad X_{0}^{*}(x)=x, \phi^{*}(t, x) \in \mathcal{R} \\
d Y_{t}^{*}(y)=-r_{t} Y_{t}^{*}(y) d t+\left(\psi^{*}(t, y)-\eta_{t}^{\mathcal{R}} Y_{t}^{*}(y)\right) . d W_{t}, \quad Y_{0}^{*}(y)=y, \quad \psi^{*}(t, y) \in \mathcal{R}^{\perp}
\end{array}\right.
$$

and assume that $\phi^{*} \in \overline{\mathcal{K}}_{\text {loc }}^{3, \delta}$, and $\psi^{*} \in \overline{\mathcal{K}}_{\text {loc }}^{2, \delta}$ for $\delta \in(0,1]$. Then, the random fields $X^{*}$ and $Y^{*}$ have the same properties as the processes of Proposition 2.8 .

Proof. Using Theorem 5.1 in the Appendix, one deduces that $X^{*}(x) \in \mathcal{K}_{\text {loc }}^{3, \varepsilon}$ and $Y^{*}(y) \in \mathcal{K}_{\text {loc }}^{2, \varepsilon}$. Then one show exactly as in [KM13] that the inverse flow $\mathcal{X}^{*}$ is a regular semimartingale.

\subsubsection{Main result concerning the reverse problem}

Let us consider two random fields, $X^{*}$ and $Y^{*}$, solution of the two SDEs (2.9) with coefficients satisfying the assumptions (2.10) of Proposition 2.8. Their properties are recalled in Proposition 2.8 and in Corollary 2.9. As denoted previously $\mathcal{X}^{*}$ is the inverse process of $X^{*}$ and $u$ is the initial utility.

The main result on the construction of consistent forward utility is obtained in two stages: the first concerns the properties of the decreasing random field $\left(U_{x}(t, x)=\right.$ $\left.Y_{t}^{*}\left(u_{x}\left(\mathcal{X}_{t}^{*}(x)\right)\right)\right)$ and of its primitive as semimartingales; the second concerns the $\mathscr{X}$ consistency of this forward utility and the optimality of the process $X^{*}$. 
Theorem 2.11 (Utility Characterization). Let us assume that the given initial utility $u$ is of class $\mathcal{C}^{3}$ and $u_{x}(x) \sim x^{-\zeta}(\zeta<1)$ in the neighborhood of $x=0$. Under the assumptions and notations of Proposition 2.8, $\left(X_{t}^{*}(x)\right)$ and $\left(Y_{t}^{*}(y)\right)$ are the unique monotonic solutions of the SDEs (2.9). Then

(i) The random field defined by $V^{*}(t, x)=Y_{t}^{*}\left(u_{x}\left(\mathcal{X}_{t}^{*}(x)\right)\right)$ is a semimartingale, integrable in the neighborhood of $x=0$, which is the derivative of a progressive utility $U(t, x)\left(V^{*}(t, x)=U_{x}(t, x)\right)$ with regular local characteristics $(\beta(t, x), \gamma(t, x))$ with

$$
\left\{\begin{array}{l}
\gamma_{x}^{\mathcal{R}}(t, x)=-\sigma^{*}(t, x) V_{x}^{*}(t, x)-V^{*}(t, x) \eta_{t}^{\mathcal{R}} \\
\gamma_{x}^{\perp}(t, x)=\vartheta^{*, \perp}\left(t, V^{*}(t, x)\right) \\
\beta(t, x)=-V^{*}(t, x) x r_{t}+\frac{1}{2} V_{x}^{*}(t, x)\left\|\sigma^{*}(t, x)\right\|^{2}
\end{array}\right.
$$

(ii) By Theorem 2.6, $\mathbf{U}$ is strongly consistent with the class $\mathscr{X}$, that is for any $X \in \mathscr{X}$, $U\left(t, X_{t}\right)$ is a supermartingale and martingale for $X_{t}^{*}$.

This result is proved in [KM13] in a SDE point of view, therefore we do not reproduce it here. A similar proof, this time in a random field point of view, is given in the context of aggregation in Theorem 3.3.

The system (2.14) can be inverted to express the characteristics of optimal processes in terms of progressive utility characteristics.

Corollary 2.12. Since $X^{*}$ and $Y^{*}$ are optimal, their characteristics $\left(\phi^{*}(t, x)\right)$ and $\left(\psi^{*}(t, y)\right)$ are explicit functionals of the progressive utility $\mathbf{U}$ and its derivatives as well as of its volatility vectors $\gamma_{x}$ along the optimal wealth process. So,

$$
\phi^{*}(t, x)=\frac{\partial_{x}\left[\gamma^{\mathcal{R}}\left(t, X_{t}^{*}(x)\right)+U\left(t, X_{t}^{*}(x)\right) \eta_{t}^{\mathcal{R}}\right]}{\left.\partial_{x}\left[U_{x}\left(t, X_{t}^{*}(x)\right)\right)\right]} \text { and } \psi^{*}\left(t, u_{x}(x)\right)=\gamma_{x}^{\perp}\left(t, X_{t}^{*}(x)\right) .
$$

\section{Aggregating multi-agents preferences}

Consider a group of agents who invest in the financial market according to their own preferences. In the following, our aim is to characterize a representative agent and his representative preference for this group. The main question is: is it possible to describe globally the behavior of all the agents by a single utility stochastic process? How could we define an aggregate utility taking into account the preferences and the sizes/weights of each agent? If all agents have the same characteristics/behaviors, then the answer is obvious. Otherwise, we classify the agents into classes with characteristics represented by the pair $\left(\mathbf{U}^{\theta}, m(d \theta)\right)$ : a consistent progressive utility and a weight.

This framework can be applied at different granularity levels. For example, one may aggregate each agent individually, that is $\left(\mathbf{U}^{\theta}, m(d \theta)\right)$ corresponds to the characteristics of one single agent. Or one can aggregate different class of agents having the same 
preferences and the same strategy inside the class (for example $\theta$ may be interpreted as the risk aversion parameter of the class and $m(d \theta)$ the proportion of this class among the whole). One may also aggregate different classes of agents who are in the same sector but who do not necessarily share the same characteristics, and whose individual characteristics are not always observable, so that one can not proceed by aggregation of each agent individually. One alternative is then to rely on a representative utility $\mathbf{U}^{\theta}$ of the sector, that is computed beforehand, using eventually different aggregation rules. Then the only information at disposal to aggregate the different sectors consists in this representative utility $\mathbf{U}^{\theta}$ of each sector and $m(d \theta)$ the relative size/weight of the sector in the whole economy.

\subsection{Aggregation of the marginal utilities}

\subsubsection{Aggregation of the initial utilities}

The global initial wealth $x$ of the economy is the sum of the individual wealths: for each $\theta$, the $\theta$-agent/class starts (at time 0 ) with a proportion $\alpha^{\theta}$ of the initial global wealth $x$ so that $x=\int \alpha^{\theta} x m(d \theta)$. At time 0 , the individual preferences $u^{\theta}$ are "dilated" into the utilities $\frac{1}{\alpha^{\theta}} u^{\theta}\left(\alpha^{\theta} x\right)$, and the global utility is the function $u(x)$,

$$
u(x)=\int \frac{1}{\alpha^{\theta}} u^{\theta}\left(\alpha^{\theta} x\right) m(d \theta), \quad \int \alpha^{\theta} m(d \theta)=1 .
$$

Technical REMARK. The measure $m(d \theta)$ can be a discrete finite measure, in this case differentiability under the integral sign is straightforward. One may also consider measures with density with respect to the Lebesgue measure. Then to ensure the 3 times-differentiability under the integral sign, locally-domination conditions are necessary: we assume that for any interval $I \subset \mathbb{R}_{+}^{*}$ there exist integrable functions $\phi_{k}^{I}(x, \theta)$ such that $\left|\partial_{x}^{k} u^{\theta}(x)\right| \leq \phi_{k}^{I}(x, \theta), \forall x \in I$ and for $k=1,2,3$. In all cases, we can pass to the limits and show $\lim _{x \rightarrow+\infty} u_{x}(x)=0$ and $\lim _{x \rightarrow 0} u_{x}(x)=+\infty$. Note that for $k=1$, since $u_{x}^{\theta}$ is by definition decreasing, it follows that for any $x_{0} \in \mathbb{R}_{+}^{*}, 0 \leq u_{x}^{\theta}(x) \leq u_{x}^{\theta}\left(x_{0}\right), \forall x \geq x_{0}$, thus it suffices to take $\phi_{k}^{\left[x_{0},+\infty[\right.}(\theta)=u_{x}^{\theta}\left(x_{0}\right)$ and assume that it is $\theta$-integrable.

Then, from (3.1), the marginal utility $u_{x}$ of the global utility is the sum of the marginal utilities, in the sense that

$$
u_{x}(x)=\int u_{x}^{\theta}\left(\alpha^{\theta} x\right) m(d \theta) .
$$

The same kind of representation holds also for the inverse function of $u_{x},-\tilde{u}_{y}$, using the correspondence between the derivatives of the utility and its dual

$$
y=\int y^{\theta}(y) m(d \theta), \quad y^{\theta}(y)=u_{x}^{\theta}\left(-\alpha^{\theta} \tilde{u}_{y}(y)\right),
$$


which leads to the remarkable feature that for any $\theta, \tilde{u}_{y}(y)=\frac{1}{\alpha^{\theta}} \tilde{u}_{y}^{\theta}\left(y^{\theta}(y)\right)$; this relation is the dual version of the $\alpha^{\theta}$-repartition of the initial wealth, $x=\frac{1}{\alpha^{\theta}}\left(\alpha^{\theta} x\right)$.

Observe that the relative risk aversion coefficient $\mathrm{R}_{\mathrm{A}}^{\mathrm{r}}(u)$ is a "probabilistic" mixture of the different risk aversion coefficients,

$$
\mathrm{R}_{\mathrm{A}}^{\mathrm{r}}(u)(x)=\frac{-x u_{x x}(x)}{u_{x}(x)}=\int \mathrm{R}_{\mathrm{A}}^{\mathrm{r}}\left(u^{\theta}\right)\left(\alpha^{\theta} x\right) \frac{u_{x}^{\theta}\left(\alpha^{\theta} x\right)}{\int u_{x}^{\theta}\left(\alpha^{\theta} x\right) m(d \theta)} m(d \theta)
$$

bounded if the family of individual risk aversion coefficients $\mathrm{R}_{\mathrm{A}}^{\mathrm{r}}\left(u^{\theta}\right)\left(\alpha^{\theta} x\right)$ is uniformly bounded in $\theta$.

\subsubsection{Aggregation of the optimal processes}

Up to the time $t$, the individuals invest optimally in a portfolio $X^{*, \theta}\left(\alpha^{\theta} x\right)$ with preferences characterized by their consistent progressive utility $\mathbf{U}^{\theta}$. It is then natural to define the global wealth in the economy at any time $t,\left(X_{t}^{*}\right)$, as the weighted sum of the individual wealths $\left(X_{t}^{*, \theta}\right)$,

$$
X_{t}^{*}(x):=\int X_{t}^{*, \theta}\left(\alpha^{\theta} x\right) m(d \theta) .
$$

Motivated by the construction of the initial utility and of its derivative $u_{x}(x)=$ $\int u_{x}^{\theta}\left(\alpha^{\theta} x\right) m(d \theta)=\int y^{\theta}\left(u_{x}(x)\right) m(d \theta)$, a natural choice is to define $Y_{t}^{*}\left(u_{x}(x)\right)$ as a mixture of individual state price processes, which is still an admissible state price process issued from $u_{x}(x)$

$$
Y_{t}^{*}\left(u_{x}(x)\right):=\int Y_{t}^{*, \theta}\left(u_{x}^{\theta}\left(\alpha^{\theta} x\right)\right) m(d \theta)=\int Y_{t}^{*, \theta}\left(y^{\theta}\left(u_{x}(x)\right)\right) m(d \theta) .
$$

Now, the problem is formulated as a reverse problem (Section 2.3) based on the increasing aggregate processes, $X_{t}^{*}(x)$ and $Y_{t}^{*}(y)$. The last difficulty is to study the regularity of those aggregate processes $X_{t}^{*}(x)$ and $Y_{t}^{*}(y)$ from the regularity of the individual processes $X_{t}^{*, \theta}(x)$ and $Y_{t}^{*, \theta}(y)$. Notice that the aggregation of processes is easier when they are considered as random fields rather as solutions of SDEs. Also, we use the representation of optimal processes given in Corollary 2.10 for the processes $X^{*, \theta}, Y^{*, \theta}$, $\left\{\begin{array}{l}d X_{t}^{*, \theta}(x)=r_{t} X_{t}^{*, \theta}(x) d t+\phi^{*, \theta}(t, x)\left(d W_{t}+\eta_{t}^{\mathcal{R}}\right), \quad X_{0}^{*, \theta}(x)=x, \phi^{*}(t, x) \in \mathcal{R} \\ d Y_{t}^{*, \theta}(y)=-r_{t} Y_{t}^{*, \theta}(y) d t+\left(\psi^{*, \theta}(t, y)-\eta_{t}^{\mathcal{R}} Y_{t}^{*, \theta}(y)\right) . d W_{t}, \quad Y_{0}^{*, \theta}(y)=y, \psi^{*}(t, y) \in \mathcal{R}^{\perp} .\end{array}\right.$

Any linear combination of portfolios $X_{t}^{*, \theta}\left(\alpha^{\theta} x\right)$ is an admissible portfolio issued from the linear combination of their initial wealth $\alpha^{\theta} x$. The same property is still true for continuous combination (under some integrability conditions). Then, the aggregate wealth process $X^{*}(x)=\int X^{*, \theta}\left(\alpha^{\theta} x\right) m(d \theta)$ is an admissible portfolio in $\mathscr{X}(x)$ and

$$
\left\{\begin{array}{l}
d X_{t}^{*}(x)=r_{t} X_{t}^{*}(x) d t+\phi^{*}(t, x) \cdot\left(d W_{t}+\eta_{t}^{\mathcal{R}} d t\right) \\
\left.\phi^{*}(t, x):=\int \phi^{*, \theta}\left(t, \alpha^{\theta} x\right)\right) m(d \theta) .
\end{array}\right.
$$


By similar arguments, the aggregate dual process $Y^{*}$ is an admissible one, with more complex dynamics, because of its dependence in $u_{x}(x)$

$$
\left\{\begin{array}{l}
d Y_{t}^{*}\left(u_{x}(x)\right)=-r_{t} Y_{t}^{*}\left(u_{x}(x)\right) d t+\left(\psi^{*}\left(t, u_{x}(x)\right)-Y_{t}^{*}\left(u_{x}(x)\right) \eta_{t}^{\mathcal{R}}\right) \cdot d W_{t} . \\
\psi^{*}\left(t, u_{x}(x)\right):=\int \psi^{*}, \theta\left(t, y_{x}^{\theta}\left(u_{x}(x)\right)\right) m(d \theta) .
\end{array}\right.
$$

Since for any $\theta, X^{*, \theta}$ and $Y^{*, \theta}$ are optimal, their characteristics are given in terms of the volatility vectors $\gamma^{\theta}$ of $\mathbf{U}^{\theta}$, which yields

$$
\left\{\begin{aligned}
\phi^{*}(t, x) & =\int\left(\frac{U_{x}^{\theta}}{U_{x x}^{\theta}}\left(\frac{\gamma_{x}^{\theta, \mathcal{R}}}{U_{x}^{\theta}}+\eta_{t}^{\mathcal{R}}\right)\right)\left(t, X_{t}^{*, \theta}\left(\alpha^{\theta} x\right)\right) m(d \theta) \\
\psi^{*}\left(t, u_{x}(x)\right) & =\int \gamma_{x}^{\theta, \perp}\left(t, X_{t}^{*, \theta}\left(\alpha^{\theta} x\right)\right) m(d \theta) .
\end{aligned}\right.
$$

\subsection{The aggregate utility}

The goal from now is to show the existence of dynamic utility $\mathbf{U}$ generating $X^{*}$ and $Y^{*}$ as optimal processes. As in the previous section, if $\mathbf{U}$ exists, then necessarily the master identity $U_{x}\left(t, X_{t}^{*}(x)\right)=Y_{t}^{*}\left(u_{x}(x)\right)$ has to be satisfied. The problem has a simple solution in the case of power utilities.

\subsubsection{Aggregating power utilities}

We come back to the standard example of power utilities and their aggregation, detailed in Paragraph 2.2.2. We assume in this subsection that not only the initial utility functions but all the progressive utilities to be aggregated are power utilities with different risk aversion coefficient.

By definition the initial utility is a mixture of dilated power utilities

$$
u(x)=\int \frac{1}{\alpha^{(\theta)}} \frac{\left(\alpha^{(\theta)} x\right)^{1-\theta}}{1-\theta} m(d \theta),
$$

which is no longer a power utility. More generally, all utility processes $\mathbf{U}^{(\theta)}$ are power utilities with constant relative risk aversion coefficient $\theta(0<\theta<1)$. As recalled in Paragraph 2.2.2, $U^{(\theta)}(t, x)=Z_{t}^{(\theta)} \frac{x^{1-\theta}}{1-\theta}$ for some process $Z^{(\theta)}$ and the optimal primal and dual processes are linear with respect to their initial conditions.

$$
X_{t}^{*,(\theta)}(x)=x \bar{X}_{t}^{*,(\theta)}, Y_{t}^{*,(\theta)}(y)=y \bar{Y}_{t}^{*,(\theta)}, \quad Z_{t}^{(\theta)}=\bar{Y}_{t}^{*,(\theta)}\left(\bar{X}_{t}^{*,(\theta)}\right)^{\theta} .
$$

The characterization of the aggregate optimal processes is easy to obtain from the definition,

$$
\begin{cases}X_{t}^{*}(x) & =x \bar{X}_{t}^{*}, \quad \bar{X}_{t}^{*}=\int \alpha^{(\theta)} \bar{X}_{t}^{*,(\theta)} m(d \theta) \\ Y_{t}^{*}\left(u_{x}(x)\right) & =\int\left(\alpha^{(\theta)} x\right)^{-\theta} \bar{Y}_{t}^{*,(\theta)} m(d \theta), \quad u_{x}(x)=\int\left(\alpha^{(\theta)} x\right)^{-\theta} m(d \theta) .\end{cases}
$$


Remark that whereas the aggregate wealth $X^{*}$ is a linear process with respect to its initial value $x$, this not true anymore for the aggregate state price density process $Y^{*}$.

The construction of a progressive utility with optimal processes $\left(x \bar{X}_{t}^{*}, Y_{t}^{*}(y)\right)$, based on the main identity $U_{x}(t, x)=Y_{t}^{*}\left(u_{x}\left(\frac{x}{\bar{X}_{t}^{*}}\right)\right)$, yields easily to the following characterization.

Proposition 3.1. The marginal utility $U_{x}(t, x)$ is the deterministic aggregation of the power marginal progressive utilities with random repartition of the optimal wealth,

$$
U_{x}(t, x)=\int \bar{Y}_{t}^{*,(\theta)}\left(\frac{\alpha^{(\theta)} x}{\bar{X}_{t}^{*}}\right)^{-\theta} m(d \theta)=\int U_{x}^{(\theta)}\left(t, \frac{\alpha^{(\theta)} \bar{X}_{t}^{*,(\theta)}}{\bar{X}_{t}^{*}} x\right) m(d \theta) .
$$

The ratio $\bar{A}_{t}^{(\theta)}=\frac{\alpha^{(\theta)} \bar{X}_{t}^{*,(\theta)}}{\bar{X}_{t}^{*}}$ is the stochastic ratio of the optimal wealths at time $t$.

As for the Pareto utility in [ILMM13], agreggating power utilities provides a family of consistent progressive utilities which is more flexible, while benefiting from some interesting features of power utilities (such as tractability).

Aggregating general consistent utilities is not as straightforward as for power utilities, and it involves the "reverse problem" techniques detailed previously in Section 2.3.

\subsubsection{The general case}

The general case will be considered as a reverse problem. Following the results in Section 2.3, some preliminary technical results are needed.

Lemma 3.2. The optimal processes $X^{*, \theta}$ and $Y^{*, \theta}$ are assumed to satisfy the regularity conditions of Theorem 2.11, with the same $\delta$ for each $\theta$ and with Lipschitz constants $C^{X, \theta}$ and $C^{Y, \theta}$ satisfying $\int C^{X, \theta} m(d \theta), \int C^{Y, \theta} m(d \theta)<\infty$. We also assume that for any interval $I \subset \mathbb{R}_{+}^{*}$ there exist integrable functions $\phi_{k}^{I}(x, \theta)$ such that $\left|\partial_{x}^{k} u^{\theta}(x)\right| \leq$ $\phi_{k}^{I}(x, \theta), \forall x \in I$ and for $k=1,2,3$. Then,

(i) There exists a constant $K$, such that for any $\theta$ and any $x, y>0, \mathbb{E}\left(X_{t}^{*, \theta}(x)\right) \leq$ $C^{X, \theta} K t x$ and $\mathbb{E}\left(Y_{t}^{*, \theta}(y)\right) \leq C^{Y, \theta} K t y$. Consequently, the integrals (3.4) and (3.5) are well defined.

(ii) The monotonic random fields $X^{*}$, defined by (3.6), is $\in \mathcal{K}_{\text {loc }}^{3, \varepsilon}$ for any $\varepsilon \in[0, \delta[$ and its inverse flow $\mathcal{X}^{*}$ is a semimartingale. Moreover $Y^{*} \in \mathcal{K}_{\text {loc }}^{2, \varepsilon}$ for any $\varepsilon \in[0, \delta[$.

(iii) $A s u_{x}^{\theta}$ is of class $\mathcal{C}^{2}(0, \infty), u_{x} \in \mathcal{C}^{2}(0, \infty)$ and the marginal utility process $U_{x}(t, x)=$ $Y_{t}^{*}\left(u_{x}\left(\mathcal{X}_{t}^{*}(x)\right)\right)$ is a $\mathcal{K}_{\text {loc }}^{2, \varepsilon}$ semimartingale for any $\varepsilon \in[0, \delta[$.

Proof. (i) is a standard result, obtained from Burkholder-Davis-Gundy and the Jensen inequalities, see [Kun97], Lemmas 4.5.3 and 4.5.5.

(ii) Combining Assumptions of this result with Theorem 5.3 leads to $X^{*, \theta} \in \mathcal{K}_{l o c}^{3, \varepsilon}$ and $Y^{*, \theta} \in \mathcal{K}_{l o c}^{2, \varepsilon}$ for any $\varepsilon \in\left[0, \delta\left[\right.\right.$. So $\sigma^{*, \theta}\left(t, X^{*, \theta}(x)\right) \in \overline{\mathcal{K}}_{l o c}^{3, \varepsilon}$ and $\vartheta^{*, \theta}\left(t, Y^{*, \theta}(y)\right) \in \overline{\mathcal{K}}_{l o c}^{2, \varepsilon}$. 
It follows that $\phi^{*}(t, x)\left(=\sigma^{*}\left(t, X_{t}^{*}(x)\right)\right)$ and $\psi^{*}(t, y)\left(=\vartheta^{*}\left(t, Y_{t}^{*}(y)\right)\right.$ are respectively in $\overline{\mathcal{K}}_{\text {loc }}^{3, \varepsilon}$ and $\overline{\mathcal{K}}_{\text {loc }}^{2, \varepsilon}$. We then conclude as in Corollary 2.10. Statement (iii) becomes obvious.

These regularity results allow us to consider the problem of consistency of the aggregate utility as a reverse problem as in Theorem 2.11.

Theorem 3.3. Under Assumptions of Lemma 3.2, $\mathbf{U}$ defined by $U(t, x)=\iint_{0}^{x} U_{x}^{\theta}\left(t, X_{t}^{*, \theta}\left(\alpha^{\theta} \mathcal{X}_{t}^{*}(z)\right)\right) d z m(d \theta) \quad$ is a consistent semimartingale progressive utility. The optimal primal and dual processes are $\left(X_{t}^{*}(x)\right)$ and $\left(Y_{t}^{*}\left(u_{x}(x)\right)=\right.$ $\left.U_{x}\left(t, X_{t}^{*}(x)\right)\right)$ and

$$
\left\{\begin{aligned}
\gamma_{x}^{\mathcal{R}}(t, x) & =-U_{x}(t, x) \eta_{t}^{\mathcal{R}}-U_{x x}(t, x) \phi^{*}\left(t, \mathcal{X}^{*}(t, x)\right) . \\
\gamma_{x}^{\perp}(t, x) & =\psi^{*}\left(t, u_{x}\left(\mathcal{X}^{*}(t, x)\right)\right) . \\
\beta(t, x) & =-r_{t} x U_{x}(t, x)+\frac{1}{2} U_{x x}(t, x)\left\|\phi^{*}\left(t, \mathcal{X}^{*}(t, x)\right)\right\|^{2} .
\end{aligned}\right.
$$

Since $\phi^{*}(t, x)=\sigma^{*}\left(t, X_{t}^{*}(x)\right)$ and $\psi^{*}(t, y)=\vartheta^{*}\left(t, Y_{t}^{*}(y)\right)$, it is easy to check the equivalence between the systems (3.9) and (2.14).

Proof. It is a consequence of Theorem 2.11, since $X^{*}$ and $Y^{*}$ satisfies respectively the SDE (2.6) and (2.7) and the regularity conditions are satisfied. We produce here the proof in this context of aggregation; the proof being still valid in the general setting of Theorem 2.11. It relies on the identity $Y_{t}^{*}\left(u_{x}(x)\right)=U_{x}\left(t, X_{t}^{*}(x)\right)$.

By statement (iii) of previous Lemma, the random field $\mathbf{U}_{x}$ is sufficiently regular to apply Itô Ventzel's formula to compute the dynamics of $U_{x}\left(t, X_{t}^{*}(x)\right)$ :

$$
\begin{aligned}
d U_{x}\left(t, X_{t}^{*}(x)\right) & =\left(\beta_{x}\left(t, X_{t}^{*}(x)\right)+\frac{1}{2} U_{x x x}\left(t, X_{t}^{*}(x)\right)\left\|\phi^{*}(t, x)\right\|^{2}\right) \\
& \left.\left.+U_{x x}\left(t, X_{t}^{*}(x)\right)\left(r_{t} X_{t}^{*}(x)+\phi^{*}(t, x)\right) \eta_{t}^{\mathcal{R}}\right)+\gamma_{x x}\left(t, X_{t}^{*}(x)\right) \phi^{*}(t, x)\right) d t \\
& +\left(\gamma_{x}\left(t, X_{t}^{*}(x)\right)+U_{x x}\left(t, X_{t}^{*}(x)\right) \phi^{*}(t, x)\right) d W_{t} .
\end{aligned}
$$

(i) By identification of the diffusion term with the one of

$$
d Y_{t}^{*}\left(u_{x}(x)\right)=-r_{t} Y_{t}^{*}\left(u_{x}(x)\right) d t+\left(\phi^{*}\left(t, u_{x}(x)\right)-Y_{t}^{*}\left(u_{x}(x)\right) \eta_{t}^{\mathcal{R}}\right) d W_{t}
$$

and by the fact that $\psi^{*}\left(t, u_{x}(x)\right)=\int \vartheta^{*, \theta}\left(t, Y_{t}^{*, \theta}\left(u_{x}^{\theta}\left(\alpha^{\theta} x\right)\right) m(d \theta)\right.$ and $\vartheta^{*, \theta}\left(t, Y_{t}^{*, \theta}\left(u_{x}^{\theta}(x)\right)=\right.$ $\gamma_{x}^{\theta, \perp}\left(t, X_{t}^{*, \theta}(x)\right)$ it follows that

$$
\gamma_{x}(t, x)+U_{x x}(t, x) \phi^{*}\left(t, \mathcal{X}^{*}(t, x)\right)=\psi^{*}\left(t, u_{x}\left(\mathcal{X}^{*}(t, x)\right)\right)-U_{x}(t, x) \eta_{t}^{\mathcal{R}}
$$

or equivalently by projecting on $\mathcal{R}$ and $\mathcal{R}^{\perp}$, 


$$
\left\{\begin{array}{l}
\gamma_{x}^{\mathcal{R}}(t, x)=-U_{x}(t, x) \eta_{t}^{\mathcal{R}}-U_{x x}(t, x) \phi^{*}\left(t, \mathcal{X}^{*}(t, x)\right) \\
\gamma_{x}^{\perp}(t, x)=\psi^{*}\left(t, u_{x}\left(\mathcal{X}^{*}(t, x)\right)\right)=\int \gamma_{x}^{\theta, \perp}\left(t, X_{t}^{*, \theta}\left(\alpha^{\theta} \mathcal{X}_{t}^{*}(x)\right)\right) m(d \theta)
\end{array}\right.
$$

(ii) Identifying the drift term, it is also easy to prove that $\mathbf{U}$ satisfies the HJB constraint (2.5). Indeed, using the characterization of $\sigma^{*}$,

$$
\begin{aligned}
\gamma_{x x}(t, x) \phi^{*}\left(t, \mathcal{X}^{*}(t, x)\right) & =\gamma_{x x}^{\mathcal{R}}(t, x) \phi^{*}\left(t, \mathcal{X}^{*}(t, x)\right) \\
& =\partial_{x}\left(U_{x x}(t, x) \phi^{*}\left(t, \mathcal{X}^{*}(t, x)\right)+U_{x}(t, x) \eta_{t}^{\mathcal{R}}\right) \phi^{*}\left(t, \mathcal{X}^{*}(t, x)\right) .
\end{aligned}
$$

It follows, after arranging the terms and identifying with the drift term of $d Y_{t}^{*, \theta}(y)$

$$
\beta_{x}(t, x)=\partial_{x}\left(-r_{t} x U_{x}(t, x)+\frac{1}{2} U_{x x}(t, x)\left\|\phi^{*}\left(t, \mathcal{X}^{*}(t, x)\right)\right\|^{2}\right)
$$

and integrating with respect to $x$ gives the HJB constraint.

\subsection{Particular case of a Pareto optimal allocation of the initial wealth}

In this work, given the wealth of each class $x^{\theta}$, we get the global wealth of the economy as $x=\int x^{\theta} m(d \theta)$. In other words, the $\alpha^{\theta}$ are imposed and given by $\alpha^{\theta}:=x^{\theta} / x$. In the literature, the approach is rather the opposite: given the global wealth of the economy $x$, the problem is to find the fair allocation of the wealth $x$ between the different classes such that the allocation is Pareto optimal, that is there are no possible alternative allocations whose realization would cause every class to gain. The Pareto optimal allocation is determined by the initial wealths $x^{*, \theta}$ with $\int x^{*, \theta} m(d \theta)=x$ such that $u(x)=\int u\left(x^{*, \theta}\right) m(d \theta)=\sup \left\{\int u\left(x^{\theta}\right) m(d \theta) \mid x^{\theta} \geq 0\right.$ and $\left.\int x^{\theta} m(d \theta)=x\right\}$. One important consequence of Pareto optimality is that the optimal pricing kernel $Y^{*, \theta}$ is the same for all agents. See for example the paper of Bank and Kramkov [BK15] that aggregates utilities parameterized by Pareto weights, for a finite number of investors, or Mrad [KM16a] for a continuum of agents and a general mixture framework. In this work, the initial repartition of the wealth is assumed to be given a priori, without reference to any "optimal allocation". The $\alpha^{\theta}$ are fixed (at time 0) and correspond to the proportion of the total wealth hold by the $\theta$-class. Therefore the aggregate utility $\mathbf{U}$ and the aggregate pricing kernel $Y^{*}$ are not standard, but they are natural candidate for aggregating different points of view of several agents, in a context without an equilibrium. It thus allows a richer class of pricing kernel that will add flexibility to capture some financial features, such that the impact of the wealth on the valuation of financial assets.

We choose to illustrate this methodology in measuring its impact on the yield curve. 


\section{Application to the yield curve}

Numerous economic issues involve the optimization of the aggregate utility of the economy. Besides, among these economic literature involving utility optimization, many papers focus on long term issues. Therefore the use of stochastic utility is particularly relevant in such modeling frameworks with long horizon. Besides, since the theoretical study of progressive utility emphasizes the dependency of the processes with respect to their initial conditions, this framework is also well adapted to study the dependency and the non-linearity of macroeconomic processes with respect to the initial value of economic indexes.

One particular example developed here consists in the valuation of long term interest rates. Modeling accurately long term interest rates is a crucial challenge in many financial topics, such as the financing of ecological project, or the pricing of longevity-linked securities or any other investment with long term impact. In the economic setting, the interest are determined endogenously at equilibrium, from the equilibrium optimal pricing kernel (see for example Vasicek [Vas77], Cox Ingersoll Ross [CIR85], Björk [Bjo12] and Piazzesi [Pia10]). The financial setting only assumes no arbitrage and with an exogenous short term interest rate, in a framework of incomplete market.

\subsection{Yield curve in incomplete market}

In the context of the high illiquidity of the bond market for longer maturities, the financial evaluation we consider is the marginal utility indifference pricing of zerocoupon bond. The link with the economic discount rate given by the Ramsey rule (in an equilibrium setting) is studied in El Karoui et al. [KHM14, KHM17b].

\subsubsection{Utility indifference pricing}

The marginal utility indifference pricing at time $t$ is not based on the "universal " market state price density $Y^{0}$ (as in complete market), but on the optimal state price density $Y^{*}(y)$ of the progressive dual utility $\widetilde{\mathbf{U}}$ of $\mathbf{U}$ (Proposition 2.5). With this new point of view, the price of some derivative $\xi_{T}$ is not given by $\pi_{0}^{0}\left(\xi_{T}\right)=\mathbb{E}\left(Y_{T}^{0} \xi_{T}\right),\left(Y_{0}^{0}=1\right)$, as in a complete market but by $\pi_{0}^{*}\left(\xi_{T}\right)(y)=\frac{1}{y} \mathbb{E}\left(Y_{T}^{*}(y) \xi_{T}\right)$, making the price depending on the global wealth $x$ of the economy via the correspondence $u_{x}(x)=y$. The pricing rule $\pi_{0}^{0}$, that is independent of the wealth, will be called market pricing rule.

DYNAMIC MARGINAL UTILITY INDIFFERENCE PRICING By definition, any state price density $\left(Y_{t}^{*}(y)\right)$ can be written as $Y_{t}^{*}(y)=Y_{t}^{0} L_{t}^{*}(y)$. All the dependencies on the wealth $x$ (or $y=u_{x}(x)$ ) is supported by the exponential martingale $L_{t}^{*}(y)$, normalized by its value at time 0 , and denoted $L_{0, t}^{*}(y):=\frac{1}{y} L_{t}^{*}(y)$. 
The marginal utility pricing rule becomes the "market pricing" of some modified payoff $\pi_{0}^{*}\left(\xi_{T}\right)(y)=\pi_{0}^{0}\left(L_{0, T}^{*}(y) \xi_{T}\right)$. The extension of the pricing rules to any date in the future is straightforward, using the conditional expectation, and the relative state price density $Y_{t, T}^{*}(y):=\frac{Y_{T}^{*}(y)}{Y_{t}^{*}(y)}$, so that

$$
\pi_{t}^{0}\left(\xi_{T}\right)=\mathbb{E}\left(Y_{t, T}^{0} \xi_{T} \mid \mathcal{F}_{t}\right) \text { and } \pi_{t}^{*}\left(\xi_{T}\right)(y)=\mathbb{E}\left(Y_{t, T}^{*}(y) \xi_{T} \mid \mathcal{F}_{t}\right)=\pi_{t}^{0}\left(L_{t, T}^{*}(y) \xi_{T}\right) .
$$

WeALth SENSitivity ANALYsis By Corollary 2.2, the volatility of $L_{0, t}^{*}(y)$ is the regular volatility random field $v_{t}^{*, \perp}(y):=\nu^{*, \perp}\left(t, Y_{t}^{*}(y)\right)=\left(Y_{t}^{*}(y)\right)^{-1} \vartheta_{t}^{*, \theta}\left(Y_{t}^{*}(y)\right)$ and

$$
\ln \left(L_{0, t}^{*}(y)\right)=\int_{0}^{t} v_{s}^{*, \perp}(y) \cdot d W_{s}-\frac{1}{2}\left\|v_{s}^{*, \perp}(y)\right\|^{2} d s .
$$

Its sensitivity in $y$ is given by

$\frac{\partial_{y} L_{0, t}^{*}(y)}{L_{0, t}^{*}(y)}=\int_{0}^{t} \partial_{y} v_{s}^{*, \perp}(y) \cdot\left(d W_{s}-v_{s}^{*, \perp}(y) d s\right)=\int_{0}^{t} \partial_{y} v_{s}^{*, \perp}(y) \cdot\left(d W_{s}+\left(\eta_{s}^{\mathcal{R}}-v_{s}^{*, \perp}(y)\right) d s\right)$.

The second equality uses the orthogonality of the vectors $v_{s}^{*, \perp}(y)$ and $\eta_{s}^{\mathcal{R}}$.

The remarkable property is that $\frac{\partial_{y} L_{0, t}^{*}(y)}{L_{0, t}^{*}(y)}$ is a martingale under the probability measure with density martingale $\Lambda_{0, t}^{*}(y)=\exp \left(\int_{0}^{t} r_{s} d s\right) Y_{0, t}^{*}(y)$ whose volatility is the $Y^{{ }^{*}}$ volatility $\left(-v_{s}^{*, \perp}(y)+\eta_{s}^{\mathcal{R}}\right)$.

Marginal Utility Bond CuRVe Applying the previous theory to the zero-coupon bond, that delivers one unit of cash at maturity $T$, we get the market bond price $B_{t}^{0}(T)$, as well as the indifference bond price $B_{t}^{*}(T, y)$ that depends on $x$ by the initial relation $y=u_{x}(x)$

$$
B_{t}^{0}(T):=\mathbb{E}\left(Y_{t, T}^{0} \mid \mathcal{F}_{t}\right), \quad \text { and } \quad B_{t}^{*}(T, y):=\mathbb{E}\left(Y_{t, T}^{*}(y) \mid \mathcal{F}_{t}\right)=\mathbb{E}\left(Y_{t, T}^{0} L_{t, T}^{*}(y) \mid \mathcal{F}_{t}\right) .
$$

a) The sensitivity of the zero-coupon bonds with respect to their maturity is interpreted in any yield market as a forward rate, that is the instantaneous short rate for an operation starting in the future at time $T$. Then, we make the distinction between market or indifference forward rate

$$
f_{t}^{0}(T):=-\partial_{T} \ln B_{t}^{0}(T), \text { respectively, } f_{t}^{*}(T, y):=-\partial_{T} \ln B_{t}^{*}(T, y) .
$$

The yield curve at current date $t$ is the function,

$$
\delta \rightarrow R_{t}^{*}(\delta, y):=\frac{1}{\delta} \int_{t}^{t+\delta} f_{t}^{*}(u, y) d u=-\frac{1}{\delta} \ln B_{t}^{*}(t+\delta) .
$$

b) The sensitivity of the bonds with respect to the initial wealth at the current date $t$ is

$$
\partial_{y} B_{t}^{*}(T, y)=\pi_{t}^{0}\left(\partial_{y} L_{t, T}^{*}(y)\right)=\mathbb{E}\left(Y_{t, T}^{*}(y) \xi_{t, T}(y) \mid \mathcal{F}_{t}\right)
$$


where $\quad-\xi_{t, T}(y)=\int_{t}^{T} \partial_{y} v_{s}^{*, \perp}(y) \cdot\left(d W_{s}+\left(\eta_{s}^{\mathcal{R}}-v_{s}^{*, \perp}(y)\right) d s\right)$. As it is often useful for financial interpretations (see Geman, El Karoui and Rochet [GEKR95]), relation (4.2) can be reinterpreted by using a change of probability measure, associated to a numeraire change,

$$
\partial_{y} B_{t}^{*}(T, y)=\mathbb{E}_{\mathbb{Q}_{(y)}^{*, T}}\left(e^{-\int_{t}^{T} r_{s} d s} \xi_{t, T}(y) \mid \mathcal{F}_{t}\right)
$$

where $\mathbb{Q}_{(y)}^{*, T}$ is the probability measure with density $\Lambda_{0, T}^{*}(y)$ and under which $d W_{s}^{*, T}=$ $d W_{s}+\left(\eta_{s}^{\mathcal{R}}-v_{s}^{*, \perp}(y)\right) d s$ is a martingale. Sometimes this probability measure is called indifference forward neutral probability.

\subsubsection{Yield curve in aggregate economy}

We come back to the framework of an economy with multi-agents having access to the same market, and so having the same market price density $Y^{0}$. They have their own progressive utilities $\mathbf{U}^{\theta}$, and then their own marginal utility pricing rules driven by their own optimal state price density $Y_{t}^{*, \theta}(y)=Y_{t}^{0} L_{t}^{*, \theta}(y)$.

Then, each agent gives a different "marginal utility price" for the zero-coupon bonds, $B_{t}^{*, \theta}(T, y)=\mathbb{E}\left(Y_{t, T}^{*, \theta}(y) \mid \mathcal{F}_{t}\right)$. In particular, the bond curves today $B_{0}^{*, \theta}\left(T, y^{\theta}\right)$ are different and a priori depend on the individual wealth $y^{\theta}$ of the agent; but a large part of the curve is explained by the common market curve $B_{0}^{0}(T)$.

AGGREGATE YIELD CURVES In the aggregate economy, the initial marginal utility is defined as a mixture of the individual marginal utilities, $u_{x}(x)=\int u_{x}^{\theta}\left(\alpha^{\theta} x\right) m(d \theta)$. Similarly, the optimal state price density $Y_{t}^{*}(y)$ is a mixture of the individual optimal state prices defined as $Y_{t}^{*}(y)=\int Y_{t}^{*, \theta}\left(y^{\theta}\right) m(d \theta)$ where $y^{\theta}\left(u_{x}(x)\right)=u_{x}^{\theta}\left(\alpha^{\theta} x\right)$.

Thanks to Equation (4.1), the bond curve $B_{t}^{*}(T, y)$ in the aggregate market satisfies $Y_{t}^{*}(y) B_{t}^{*}(T, y)=\mathbb{E}\left(Y_{T}^{*}(y) \mid \mathcal{F}_{t}\right)=\int \mathbb{E}\left(Y_{T}^{*, \theta}\left(y^{\theta}\right) \mid \mathcal{F}_{t}\right) m(d \theta)=\int B_{t}^{*, \theta}(T, y) Y_{t}^{*, \theta}\left(y^{\theta}\right) m(d \theta)$.

The aggregate bond curve is a mixture of different bond curves with respect to the non normalized densities $Y_{t}^{*, \theta}\left(y^{\theta}\right)$, whose integral is by definition $Y_{t}^{*}(y)$.

It is easy to take the derivative in maturity in the previous equality, and to use intensively that $\partial_{T} B_{t}^{*}(T, y)=-f_{t}^{*}(T, y) B_{t}^{*}(T, y)$ where $f_{t}^{*}(T, y)$ is the instantaneous forward rate in the aggregate market. Thus we obtain that

$$
f_{t}^{*}(T, y) Y_{t}^{*}(y) B_{t}^{*}(T, y)=\int f_{t}^{*, \theta}(T, y)\left(Y_{t}^{*, \theta}\left(y^{\theta}\right) B_{t}^{*, \theta}(T, y)\right) m(d \theta) .
$$

The remarkable feature of these two decompositions is that the non normalized mixing processes may be chosen to be martingales:

- It is obvious in the case of spot forward rates where the mixing processes are $Y_{t}^{*, \theta}\left(y^{\theta}\right) B_{t}^{*, \theta}\left(T, y^{\theta}\right)$ which are by definition the exponential martingales determining the volatility of the bond. 
- For the mixing of the bonds, the non normalized coefficients $Y_{t}^{*, \theta}\left(y^{\theta}\right)=Y_{t}^{0} L_{t}^{*, \theta}\left(y^{\theta}\right)$ (having the common factor $\left.Y_{t}^{0}\right)$ can be replaced by the martingales $L_{t}^{*, \theta}\left(y^{\theta}\right)$ without change after renormalization.

All these results are gathered in the next proposition:

Proposition 4.1. In an aggregate economy,

(i) The marginal utility bond curve $B_{t}^{*}(T, y)$ is a normalized mixture of individual bond curves, based on the martingales $L_{t}^{*, \theta}$,

$$
B_{t}^{*}(T, y)=\int B_{t}^{*, \theta}\left(T, y^{\theta}\right) \frac{L_{t}^{*, \theta}\left(y^{\theta}\right) m(d \theta)}{\int L_{t}^{*, \theta}\left(y^{\theta}\right) m(d \theta)} .
$$

(ii) The marginal utility spot forward rates $f_{t}^{*}(T, y)$ is a normalized mixture of individual spot forward rates curve based on the martingales $Y_{t}^{*, \theta}\left(y^{\theta}\right) B_{t}^{*, \theta}\left(T, y^{\theta}\right)$

$$
f_{t}^{*}(T, y)=\int f_{t}^{*, \theta}\left(T, y^{\theta}\right) \frac{B_{t}^{*, \theta}\left(T, y^{\theta}\right) L_{t}^{*, \theta}\left(y^{\theta}\right)}{\int B_{t}^{*, \theta}\left(T, y^{\theta}\right) L_{t}^{*, \theta}\left(y^{\theta}\right) m(d \theta)} m(d \theta) .
$$

Indifference Bonds pricing for power utilities We come back to the framework of aggregate power utilities, that will be used in the forthcoming numerical application in Section 4.2. We consider $N$ agents with consistent power utilities characterized by their relative risk aversion parameters $\theta_{1}<\cdots<\theta_{N}$, as studied in Paragraph 2.2.2. Then, their optimal state prices $Y_{t}^{*, \theta}(y)$ are linear in $y$ with coefficient $\bar{Y}_{t}^{*, \theta}$, and the individual price of zero-coupon bonds with maturity $T$ does not depend on $y$ and more generally, $\bar{B}_{t}^{*, \theta}(T)=\mathbb{E}\left(\bar{Y}_{t, T}^{*, \theta} \mid \mathcal{F}_{t}\right)$. The aggregate indifference zero-coupon price $\bar{B}_{0}^{*}(T, y)$, computed at time 0 for simplicity, is given by

$$
\bar{B}_{0}^{*}(T, y)=\frac{\sum_{i=1}^{N} y^{\theta_{i}}(y) \bar{B}_{0}^{*, \theta_{i}}(T)}{y}=\frac{\sum_{i=1}^{N}\left(\alpha_{i} x\right)^{-\theta_{i}} \bar{B}_{0}^{*, \theta_{i}}(T)}{\sum_{i=1}^{N}\left(\alpha_{i} x\right)^{-\theta_{i}}}
$$

with $y=\sum_{i=1}^{N} y^{\theta_{i}}(y)=u_{x}(x)$ and for power utilities, $y^{\theta_{i}}(y)=u_{x}^{\theta_{i}}\left(\alpha_{i} x\right)=\left(\alpha^{i} x\right)^{-\theta_{i}}$. Asymptotic BeHAVIor Using the linearity of the optimal state prices $Y_{t}^{*, \theta}\left(y^{\theta_{i}}\right)$ in $y^{\theta_{i}}$ and the form of the marginal initial power utilities $u_{x}^{\theta_{i}}$, the asymptotic behavior of the aggregate zero-coupon price, for $y$ around 0 (respectively $\infty$ ), is straightforward and relies on the convergence of the random measure $\frac{\sum_{i=1}^{N} y^{\theta_{i}}(y) \delta_{\theta_{i}}}{y}$ towards a dirac measure that charges the agent with the smallest (respectively the largest) risk aversion $\theta_{i}$. Indeed, scaling the initial wealth $x$ with a factor $\lambda \in \mathbb{R}^{+}$, leads to the following asymptotics (for $\lambda \rightarrow 0$ or $\infty$ )

$$
\lim _{y \rightarrow 0} B_{0}^{*}(T, y)=B_{0}^{\theta_{1}}(T) \text { and } \lim _{y \rightarrow+\infty} B_{0}^{*}(T, y)=B_{0}^{\theta_{N}}(T) .
$$

This means that, when the wealth tends to infinity, the aggregate zero-coupon price converges to the one priced by the less risk averse agent, whereas when the wealth tends 
to zero, it converges to the one priced by the more risk averse agent. This is a similar result as the ones stated in Cvitanic, Jouini et al. [CJMN11].

\subsection{Numerical Results}

The numerical illustration is developed in the simple model of an economy where three agents invest in an incomplete market with two independent Brownian motions: the market is characterized by the market state price density $Y^{0}$ with a constant market risk premium $(\eta, 0)$ and a stochastic interest rate. The volatility vector of admissible portfolios only depends on the first component $\kappa_{t}=\left(\kappa_{t}^{1}, 0\right)$; the optimal dual orthogonal volatility $\left(0, v^{*, \theta}\right)$ is also assumed to be constant in time and independent of $y$, where $\theta \in] 0,1[$ is the relative risk aversion parameter characterizing the agent starting with a power utility:

$$
Y_{t}^{*, \theta}(y)=y e^{-\int_{0}^{t} r_{s} d s} e^{-\eta W_{t}^{1}+v^{*, \theta} W_{t}^{2}-\frac{1}{2}\left(\eta^{2}+\left(v^{*, \theta}\right)^{2}\right) t}
$$

We also need to specify a model for the spot rate common for all agents. The simplest and currently used in financial market is the Vasicek model [Vas77].

VASICEK MOdEL FOR the SPOT RATE $r_{t}$ : We assume a Vasicek model for the spot rate $r_{t}$,

$$
d r_{t}=a\left(b-r_{t}\right) d t+\sigma_{1} d W_{t}^{1}+\sigma_{2} d W_{t}^{2},
$$

which is a Gaussian Ornstein-Uhlenbeck process given by

$$
r_{t}=r_{0}+b e^{-a t}+\int_{0}^{t} e^{-a(t-s)}\left(\sigma_{1} d W_{t}^{1}+\sigma_{2} d W_{t}^{2}\right)
$$

The market zero-coupon bond is given from the market state price density $Y_{t}^{0}$, by $B_{t}^{0}(T)=\mathbb{E}\left(Y_{t, T}^{0} \mid \mathcal{F}_{t}\right)$. From Gaussian standard calculus, it is well-known that the market yield curve $R_{t}^{0}(\delta)=-\frac{1}{\delta} \ln B_{t}^{0}(t+\delta)$ has the following form

$$
\begin{gathered}
R_{t}^{0}(\delta)=R_{\infty}^{0}-\left(R_{\infty}^{0}-r_{t}\right) \frac{\left(1-e^{-a \delta}\right)}{a \delta}+\frac{\mathfrak{s}^{2, r}}{a^{2}} \frac{\left(1-e^{-a \delta}\right)^{2}}{4 a \delta} \\
\text { where } R_{\infty}^{0}=b-\frac{1}{2}\left[\frac{\mathfrak{s}^{2, r}}{a^{2}}+2 \frac{\sigma_{1} \eta}{a}\right] \text { and } \mathfrak{s}^{2, r}=\left(\sigma_{1}\right)^{2}+\left(\sigma_{2}\right)^{2} .
\end{gathered}
$$

Indifference Yield CURVE In this example, the indifference yield curve is obtained by multiplication of the market price density $\left(Y_{t}^{0}\right)$ by the exponential martingale $L_{t}^{*, \theta}=$ $\exp \left(v^{*, \theta} W_{t}^{2}-\frac{1}{2}\left(v^{*, \theta}\right)^{2} t\right)$ which depends on the Brownian motion $W^{2}$ only.

The bonds are obtained as previously, using the probability measure $\mathbb{Q}^{\perp, \theta}=L_{T}^{*, \theta} \cdot \mathbb{P}$ in place of $\mathbb{P}$. Under $\mathbb{Q}^{\perp, \theta}, W^{1}$ is still a Brownian motion but $W^{2}$ becomes $W_{t}^{2}=W_{t}^{2, \perp, \theta}+$ $v^{*, \theta} t$ where $W^{2, \perp, \theta}$ is a $\mathbb{Q}^{\perp, \theta}$-Brownian motion. The spot rate $r_{t}$ remains an OrnsteinUhlenbeck process under $\mathbb{Q}^{\perp, \theta}$, only the level $b$ is modified into $b^{\perp, \theta}=b+\frac{1}{a} \sigma_{2} v^{*, \theta}$. This 
modification has an impact on the infinite rate, that becomes $R_{\infty}^{*, \theta}=R_{\infty}^{0}+\frac{1}{a} \sigma_{2} v^{*, \theta}$. The new yield curve is now:

$$
\left\{\begin{array}{l}
R_{t}^{*, \theta}(\delta)=R_{\infty}^{*, \theta}-\left(R_{\infty}^{*, \theta}-r_{t}\right) \frac{\left(1-e^{-a \delta}\right)}{a \delta}+\frac{\mathfrak{5}^{2, r}}{a^{2}} \frac{\left(1-e^{-a \delta}\right)^{2}}{4 a \delta} \\
R_{t}^{*, \theta}(\delta)=R_{t}^{0}(\delta)-\sigma_{2} v^{*, \theta}\left(\frac{1-e^{-a \delta}-a \delta}{a^{2} \delta}\right) .
\end{array}\right.
$$

The same kind of equation holds for the different forward rates. In particular the spread between the indifference curve and the market curve is given by:

$$
f_{t}^{*, \theta}(T)-f_{t}^{0}(T)=-\sigma_{2} v^{*, \theta}\left(\frac{1-e^{-a(T-t)}}{a}\right) .
$$

AGREGGATE BOND CURVE For the aggregate bond curve, we consider the aggregation of three agents with power utility and risk aversion parameter $\left(\theta_{i}\right)$ and with a given initial repartition of the wealth $\left(\alpha_{i}\right)$ (see Section 3.2.1). In this case, the zero-coupon bond is evaluated as

$$
B_{t}^{*}(T, x, \alpha)=\frac{\sum_{i=1}^{3}\left(\alpha_{i} x\right)^{-\theta_{i}} B_{t}^{*, \theta_{i}}(T)}{u_{x}(x)}
$$

where $x$ and $\alpha$ stand here to remind that the aggregate price depends on the initial wealth and on the initial choice of the parameters $\left(\alpha_{i}\right)$.

The ratio $\frac{B_{t}^{*}(T, x, \alpha)}{B_{t}^{0}(T)}$ is particularly simple, using the notation $\zeta_{2}(T-t)=\sigma_{2}\left(\frac{1-e^{-a(T-t)}-a(T-t)}{a^{2}}\right)$

$$
\frac{B_{t}^{*}(T, x, \alpha)}{B_{t}^{0}(T)}=\frac{\sum_{i=1}^{3}\left(\alpha_{i} x\right)^{-\theta_{i}} \exp \left(-\zeta_{2}(T-t) v^{*, \theta_{i}}\right)}{u_{x}(x)}=\frac{\sum_{i=1}^{3} \exp \left(-\theta_{i} \ln \left(\alpha_{i} x\right)-\zeta_{2}(T-t) v^{*, \theta_{i}}\right)}{u_{x}(x)} .
$$

Simulations The following simulations are provided taking $v^{*, \theta}=\theta v^{*}$ for some given constant $v^{*}$. For any $\theta$, the individual yield curve $\delta \mapsto R_{0}^{*, \theta}(\delta)$ does not depend on the wealth and is a Vasicek curve with infinite rate $R_{0}^{*, \theta}(\delta)=R_{0}^{0}(\delta)-\sigma_{2} \theta v^{*}\left(\frac{1-e^{-a \delta}-a \delta}{a^{2} \delta}\right)$. In the figures we choose the following numerical values for the parameters

$$
r_{0}=5 \%, a=1, b=0.2, \sigma_{1}=20 \%, \sigma_{2}=15 \%, \eta=20 \%, v^{*}=80 \% .
$$

It provides a standard increasing yield curve, but Vasicek model can also achieve others forms of curve (not monotonic and with bumps) for other parameters values.

In Figure 1 we draw the individual yield curve $R_{0}^{*, \theta}(\delta)$ of each class (Vasicek yield curves), for different values of $\theta$. 


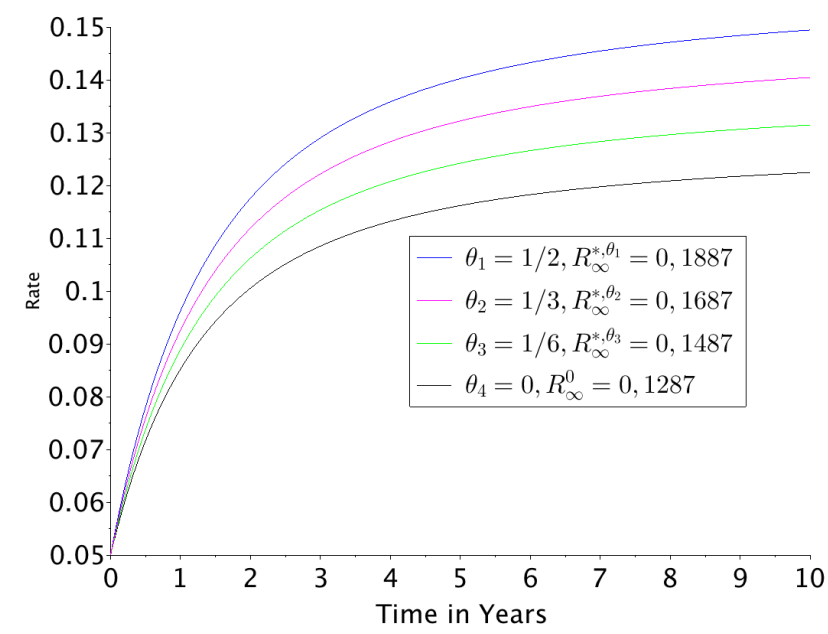

Figure 1: Individual yield curve $R_{0}^{*, \theta}(\delta)$ for different values of $\theta$

From now on, we will represent only the spreads between the different rate curves and the market yield curve $R_{0}^{0}(\delta)$, namely $R_{0}^{*, \theta}(\delta)-R_{0}^{0}(\delta)$. Figure 2 represents the spread of the individual curve for three different values of $\theta$ as well as the spread of the aggregate curve. The spread of the aggregate curve depends on $x$ and the $\alpha_{i}$, we choose here $x=10, \alpha_{1}=1 / 8, \alpha_{2}=1 / 2, \alpha_{3}=3 / 8$ (unless other numerical values are specified).

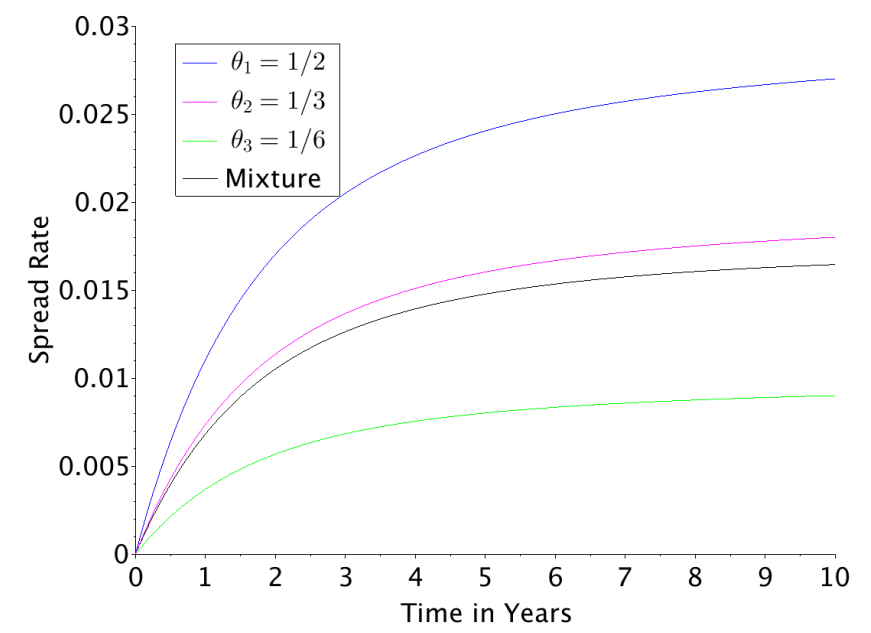

Figure 2: Individual and aggregate yield curve spread

Figure 3 (respectively Figure 4) illustrates the sensitivity of the aggregate yield curve on the initial wealth $x$ available on the market (respectively on the initial proportion parameters $\alpha^{\theta}$ ). 


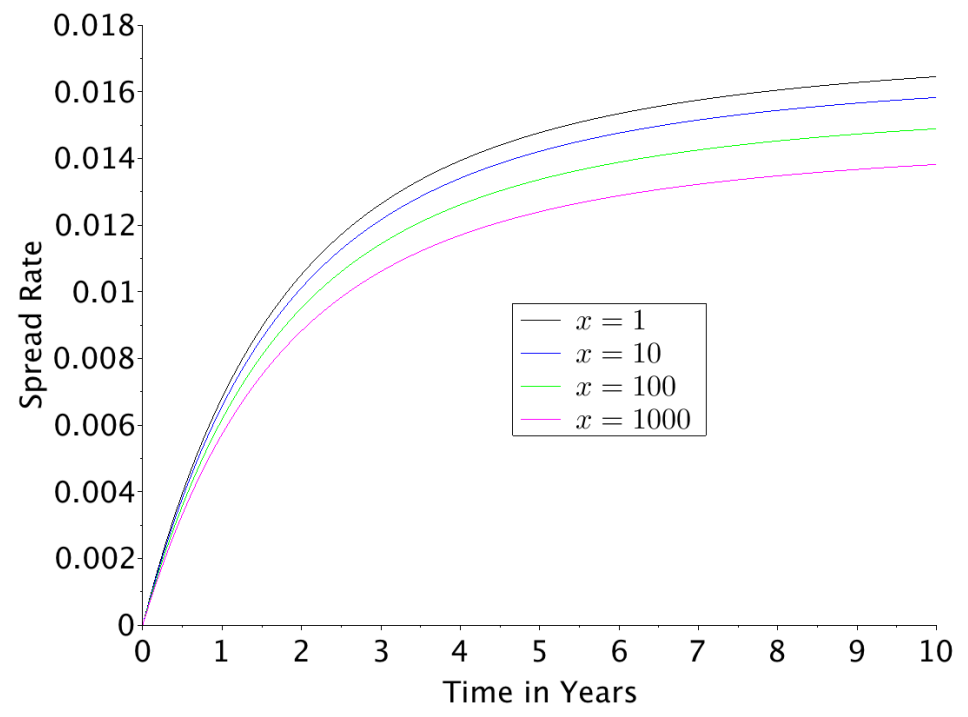

Figure 3: Aggregate yield curve spread depending of the wealth $x$

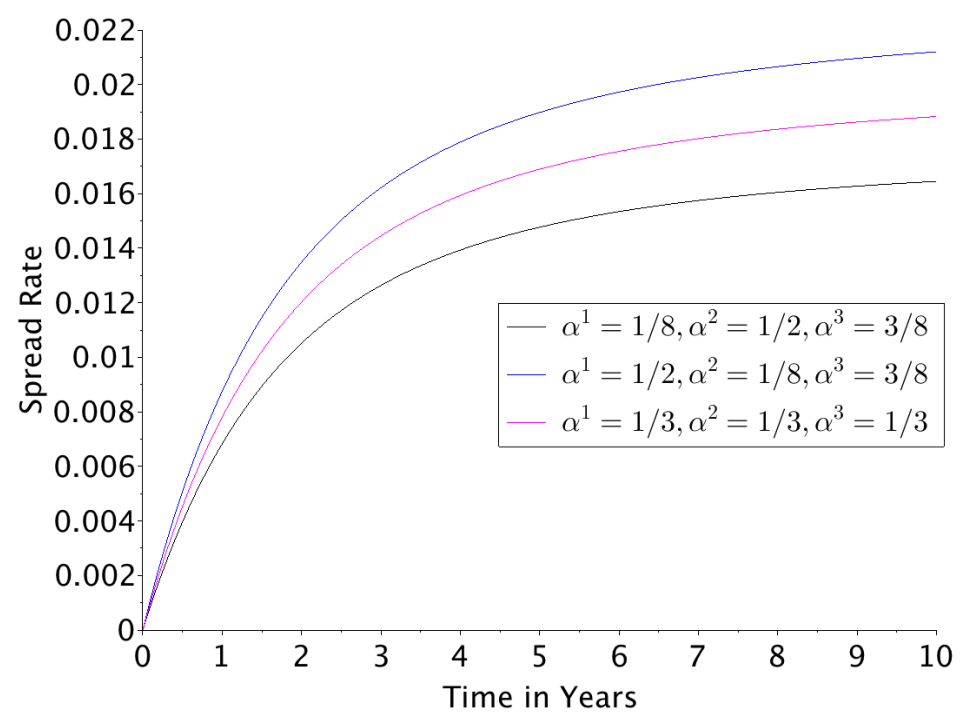

Figure 4: Aggregate yield curve spread depending on the initial proportion parameters $\alpha$ 


\section{Appendix}

Itô-Ventzel's formula In this paper we used the Itô-Ventzel formula that gives the decomposition of the compound random field $G\left(t, X_{t}\right)$ for $G(t, x)=G(0, x)+$ $\int_{0}^{t} \phi(s, x) d s+\int_{0}^{t} \psi(s, x) \cdot d W_{s}$ regular enough (see Theorem 5.1 below) and for any Itô semimartingale $X$. This decomposition is the sum of three terms: the first one is the "differential in $t$ " of $G$, the second one is the classic Itô's formula (without differentiation in time) and the third one is the infinitesimal covariation between the martingale part of $G_{x}$ and the martingale part of $X$, all these terms being taken in $X_{t}$.

$$
\begin{aligned}
d G\left(t, X_{t}\right) & =\left(\phi\left(t, X_{t}\right) d t+\psi\left(t, X_{t}\right) \cdot d W_{t}\right) \\
& +\left(G_{x}\left(t, X_{t}\right) d X_{t}+\frac{1}{2} G_{x x}\left(t, X_{t}\right) d<X, X>_{t}\right)+\left(<\psi_{x}\left(t, X_{t}\right) d W_{t}, d X_{t}>\right) .
\end{aligned}
$$

When $G$ has only finite variation, the formula is reduced to a classic Itô's formula, since in this case $\psi(t, x) \equiv 0, \phi\left(t, X_{t}\right)=\partial_{t} G_{t}\left(t, X_{t}\right)$.

Different spaces of regular random fields We give here the regularity conditions needed in Theorem 2.11 to characterize a consistent progressive utility from its optimal processes. These regularity conditions are related to the SDEs' coefficients.. Let $(\phi, \psi)$ be continuous $\mathbb{R}^{k}$-valued progressive random fields and let $m$ be a nonnegative integer, and $\delta$ a number in $(0,1]$. We need to control the asymptotic behavior in 0 and $\infty$ of $\phi$ and $\psi$, and the regularity of their Hölder derivatives (when they exist). More precisely, let $\phi \in \mathcal{C}^{m, \delta}(] 0,+\infty[)$ be $(m, \delta)$-times ${ }^{3}$ continuously differentiable in $x$ for any $t$, a.s.

For any subset $K \subset] 0,+\infty$ [, we define the family of random (Hölder) $K$-semi-norms

$$
\left\{\begin{array}{l}
\|\phi\|_{m: K}(t, \omega)=\sup _{x \in K} \frac{\|\phi(t, x, \omega)\|}{x}+\sum_{1 \leq j \leq m} \sup _{x \in K}\left\|\partial_{x}^{j} \phi(t, x, \omega)\right\| \\
\|\psi\|_{m, \delta: K}(t, \omega)=\|\psi\|_{m: K}(t, \omega)+\sup _{x, y \in K} \frac{\left\|\partial_{x}^{m} \psi(t, x, \omega)-\partial_{x}^{m} \psi(t, y, \omega)\right\|}{|x-y|^{\delta}} .
\end{array}\right.
$$

When $K$ is all the domain $] 0,+\infty\left[\right.$, we simply write $\|\cdot\|_{m}(t, \omega)$, or $\|\cdot\|_{m, \delta}(t, \omega)$.

Calligraphic notation recalls that these semi-norms are random.

a) $\mathcal{K}_{l o c}^{m, \delta}$ (resp. $\overline{\mathcal{K}}_{l o c}^{m, \delta}$ ) denotes the set of all $\mathcal{C}^{m, \delta}$-random fields such that for any compact $K \subset] 0,+\infty\left[\right.$, and any $T, \int_{0}^{T}\|\phi\|_{m, \delta: K}(t, \omega) d t<\infty,\left(\operatorname{resp} \cdot \int_{0}^{T}\|\psi\|_{m, \delta: K}^{2}(t, \omega) d t<\infty\right)$.

b) When these different norms are well-defined on the whole space $] 0,+\infty[$, we use the notations $\mathcal{K}_{b}^{m}, \overline{\mathcal{K}}_{b}^{m}$ or $\mathcal{K}_{b}^{m, \delta}, \overline{\mathcal{K}}_{b}^{m, \delta}$.

\footnotetext{
${ }^{3}$ That is $\phi$ is $m$-times continuously differentiable with $\phi^{(m)}$ being $\delta$-Hölder
} 
Differentiability of Itô random fields We discuss the regularity of an Itô semimartingale random field

$$
G(t, x)=G(0, x)+\int_{0}^{t} \phi(s, x) d s+\int_{0}^{t} \psi(s, x) \cdot d W_{s}
$$

in connection with the regularity of its local characteristics $(\phi, \psi)$. An Itô random field $\mathbf{G}$ is said to be a $\mathcal{K}_{l o c}^{m, \delta}$-semimartingale, whenever $G(0, x)$ is of class $\mathcal{C}^{m, \delta}, B^{G}(t, x)=$ $\int_{0}^{t} \phi(s, x) d s$ is of class $\mathcal{K}_{l o c}^{m, \delta}$, and $M^{G}(t, x)=\int_{0}^{t} \psi(s, x) . d W_{s}$ is of class $\overline{\mathcal{K}}_{l o c}^{m, \delta}$. As in Kunita [Kun97], we are concerned with the regularity of $\mathbf{G}$ (the regularity of its local characteristics $(\phi, \psi)$ being given) and conversely with the regularity of $(\phi, \psi)$ (the regularity of $\mathbf{G}$ being given). Theorem 3.1.2, Theorem 3.1.3 and Theorem 3.3.3 in [Kun97] give the differential rules (term by term) of the dynamics of an Itô random field and the minimal condition to apply Itô-Ventzel's formula.

Theorem 5.1 (Differential Rules). Let $\delta \in(0,1]$ and $\mathbf{G}$ be an Itô semimartingale random field with local characteristics $(\phi, \psi), G(t, x)=G(0, x)+\int_{0}^{t} \phi(s, x) d s+\int_{0}^{t} \psi(s, x) . d W_{s}$ (i) If $\mathbf{G}$ is a $\mathcal{K}_{\text {loc }}^{m, \delta}$-semimartingale for some $m \geq 0$, its local characteristics $(\phi, \psi)$ are of class $\mathcal{K}_{\text {loc }}^{m, \varepsilon} \times \overline{\mathcal{K}}_{\text {loc }}^{m, \varepsilon}$ for any $\varepsilon<\delta$, and conversely.

(ii) Conversely, if the local characteristics $(\phi, \psi)$ are of class $\mathcal{K}_{\text {loc }}^{m, \delta} \times \overline{\mathcal{K}}_{\text {loc }}^{m, \delta}$, then $\mathbf{F}$ is a $\mathcal{K}_{\text {loc }}^{\text {m, }}$-semimartingale for any $\varepsilon<\delta$.

(iii) For $m \geq 1$, the derivative random field $\mathbf{G}_{\mathbf{X}}$ is an Itô random field with local characteristics $\left(\phi_{x}, \psi_{x}\right)$, and for $m \geq 2$ the Itô-Ventzel formula is applicable.

(iv) Moreover, if $\mathbf{G}$ is a $\mathcal{K}_{\text {loc }}^{1, \delta} \cap \mathcal{C}^{2}$-semimartingale, for any Itô process $X, G(., X$.) is a continuous Itô semimartingale satisfying the Itô-Ventzel formula (5.1).

Differentiability of SDEs solutions It is well known on the SDE's theory that is sufficient (but not necessary) to take a coefficients $(\mu, \sigma) \in \mathcal{K}_{b}^{0,1}, \overline{\mathcal{K}}_{b}^{0,1}$ to ensure the existence of monotonic global (non-explosive) solution of $\operatorname{SDE}(\mu, \sigma)$ with range $[0, \infty)$ and a good behavior near to zero and infinity (see the discussion in [KM13] or Kunita's book [Kun97]). Otherwise, local regularity on SDEs coefficients appears as a kind of minimal assumption to ensure the regularity of a global solution if there exists.

Definition 5.2. A $S D E(\mu, \sigma)$ is said to be of class $\mathcal{S}^{m, \delta}$ if

a) the coefficients $(\mu, \sigma)$ are in the spaces $\left(\mathcal{K}_{\text {loc }}^{m, \delta}, \overline{\mathcal{K}}_{\text {loc }}^{m, \delta}\right)$

b) the maximal solution $X$ is non explosive.

Classical examples of $\mathcal{S}^{m, \delta} \operatorname{SDEs}$ are given by $\operatorname{SDE}(\mu, \sigma)$ when $(\mu, \sigma)$ are in the spaces $\left(\mathcal{K}_{b}^{m}, \overline{\mathcal{K}}_{b}^{m}\right)$, or even in $\left(\mathcal{K}_{b}^{0}, \overline{\mathcal{K}}_{b}^{0}\right) \cap\left(\mathcal{K}_{\text {loc }}^{m, \delta}, \overline{\mathcal{K}}_{\text {loc }}^{m, \delta}\right)$.

Theorem 5.3 (Flows property of SDE). We consider the $S D E(\mu, \sigma)$

$$
d X_{t}=\mu\left(t, X_{t}\right) d t+\sigma\left(t, X_{t}\right) \cdot d W_{t}, \quad X_{0}=x .
$$


Let $m \geq 1, \delta \in(0,1]$ and $\varepsilon<\delta$.

(i) Assume uniformly Lipschitz coefficients, that is $(\mu, \sigma) \in \mathcal{K}_{\mathbf{b}}^{0,1} \times \overline{\mathcal{K}}_{\mathbf{b}}^{0,1}$. Then, (5.4) admits a unique strong solution $X$ which is strictly monotonic satisfying $X(0)=0$ and $X(+\infty):=\lim _{x \rightarrow+\infty} X(x)=+\infty$.

(ii) Assume $\mu \in \mathcal{K}_{\mathbf{b}}^{m, \delta}$ and $\sigma \in \overline{\mathcal{K}}_{\mathbf{b}}^{m, \delta}$.

a) Then the solution $\mathbf{X}=\left(X_{t}(x), x>0\right)$ is a $\mathcal{K}_{\text {loc }}^{m, \varepsilon}$ semimartingale the derivatives $\mathbf{X}_{\mathbf{x}}$ and $\mathbf{1} / \mathbf{X}_{\mathbf{x}}$ are $\mathcal{K}_{\text {loc }}^{m-1, \varepsilon}$-semimartingales. Its inverse $\mathbf{X}^{-\mathbf{1}}$ is also of class $\mathcal{C}^{m}$.

b) The local characteristics of $\mathbf{X}, \lambda^{X}(t, x)=\mu\left(t, X_{t}(x)\right)$ and $\theta^{X}(t, x)=\sigma\left(t, X_{t}(x)\right)$ have only local properties and belong to $\mathcal{K}_{\text {loc }}^{m, \varepsilon} \times \overline{\mathcal{K}}_{\text {loc }}^{m, \varepsilon}$.

\section{References}

[Bjo12] T. Bjork. Equilibrium theory in continuous time. Lecture Notes, 2012. 19

[BK15] Peter Bank and Dmitry Kramkov. A model for a large investor trading at market indifference prices. ii: continuous-time case. The Annals of Applied Probability, 25(5):2708-2742, 2015. 18

[CIR85] J.C. Cox, J.C. Ingersoll, and S.A. Ross. A theory of the term structure of interest rates. Econometrica, 53(2):385-403, March 1985. 3, 19

[CJMN11] Jakša Cvitanić, Elyes Jouini, Semyon Malamud, and Clotilde Napp. Financial markets equilibrium with heterogeneous agents. Review of Finance, 16(1):285-321, 2011. 2, 23

[CK02] Yeung Lewis Chan and Leonid Kogan. Catching up with the joneses: Heterogeneous preferences and the dynamics of asset prices. Journal of Political Economy, 110:1255-1285, 2002. 2

[Dum89] Bernard Dumas. Two-person dynamic equilibrium in the capital market. The Review of Financial Studies, 2(2):157-188, 1989. 2

[FP09] D. Filipovic and E. Platen. Consistent Market Extensions under the Benchmark Approach. Mathematical Finance, 19(1):41-52, 2009. 5

[GEKR95] Helyette Geman, Nicole El Karoui, and Jean-Charles Rochet. Changes of numeraire, changes of probability measure and option pricing. Journal of Applied probability, 32(2):443-458, 1995. 21

[ILMM13] Masako Ikefuji, Roger JA Laeven, Jan R Magnus, and Chris Muris. Pareto utility. Theory and Decision, 75(1):43-57, 2013. 16 
[JN10] Elyès Jouini and Clotilde Napp. Unbiased disagreement in financial markets, waves of pessimism and the risk-return trade-off. Review of Finance, 15(3):575-601, 2010. 2

[KGR95] N. El Karoui, H. Geman, and J.C. Rochet. Changes of numeraire, changes of probability measure and option pricing. J. Appl. Prob., 32(2):443-458, 1995. 5

[KHM14] N. El Karoui, C. Hillairet, and M. Mrad. Affine Long Term Yield Curves : an application of the Ramsey Rule with Progressive Utility. Journal of Financial Engineering, Vol. 1, No. 1, 2014. 19

[KHM17a] N. El Karoui, C. Hillairet, and M. Mrad. Consistent utility of investment and consumption : a forward/backward spde viewpoint. Stochastics., 2017. 2,6

[KHM17b] N. El Karoui, C. Hillairet, and M. Mrad. Ramsey rule with forward/backward utility for long term yield curves modeling. Preprint, 2017. 19

[KLS87] I. Karatzas, J.P. Lehoczky, and S.E. Shreve. Optimal portfolio and consumption decisions for a small investor on a finite horizon. SIAM J. Control and Optimization, 25:1557-1586, 1987. 4

[KM13] N. El Karoui and M. Mrad. An Exact connection between two Solvable SDEs and a Non Linear Utility Stochastic PDEs. SIAM Journal on Financial Mathematics,, 4(1):697-736, 2013. 3, 6, 7, 8, 9, 10, 11, 12, 28

[KM16a] N. El Karoui and M. Mrad. Mixture of consistent stochastic utilities, and a priori randomness. Preprint., 2016. 18

[KM16b] N. El Karoui and M. Mrad. Stochastic utilities with a given optimal portfolio : approach by stochastic flows. Preprint., 2016. 3

[KS99] D. Kramkov and W. Schachermayer. The asymptotic elasticity of utility functions and optimal investment in incomplete markets. Annals of Applied Probability, 9(3):904-950, 1999. 6

[KS01] I. Karatzas and S.E. Shreve. Methods of Mathematical Finance. Springer, September 2001. 4 
[KS03] D. Kramkov and W. Schachermayer. Necessary and sufficient conditions in the problem of optimal investment in incomplete markets. Annals of Applied Probability, 13(4):1504-1516, 2003. 4

[Kun97] H. Kunita. Stochastic flows and stochastic differential equations, volume 24 of Cambridge Studies in Advanced Mathematics. Cambridge University Press, Cambridge, 1997. Reprint of the 1990 original. 11, 16, 28

[PH06] E. Platen and D. Heath. A benchmark approach to quantitative finance. Springer Finance. Springer-Verlag, Berlin, 2006. 5

[Pia10] Monika Piazzesi. Affine term structure models. Handbook of financial econometrics, 1:691-766, 2010. 19

[Rog03] LCG Rogers. Duality in constrained optimal investment and consumption problems: a synthesis. In Paris-Princeton Lectures on Mathematical Finance 2002, pages 95-131. Springer, 2003. 7

[Vas77] Oldrich Vasicek. An equilibrium characterization of the term structure. Journal of financial economics, 5(2):177-188, 1977. 3, 19, 23

[Wan96] Jiang Wang. The term structure of interest rates in a pure exchange economy with heterogeneous investors. Journal of Financial Economics, 41(1):75 - 110, 1996. 2

[Yan10] Hongjun Yan. Is noise trading cancelled out by aggregation? Management Science, 56(7):1047-1059, 2010. 2 